ARTICLE

DOI: $10.1038 / \mathrm{s} 41467-018-05142-3$

\title{
Dynamic and programmable morphology and size evolution via a living hierarchical self-assembly strategy
}

Xing Wang ${ }^{1}$ Peiyuan Gao 2 , Yanyu Yang ${ }^{1,3}$, Hongxia Guo ${ }^{1,4} \&$ Decheng Wu ${ }^{1,4}$

Recent advances in the preparation of shape-shifting and size-growing nanostructures are hot topics in development of nanoscience, because many intelligent functions are always relied on their shape and dimension. Here we report a tunable manipulation of sequential selfassembled transformation in situ via a hierarchical assembly strategy based on a living thiol-disulfide exchange reaction. By tailoring the external stimuli, the reactive points can be generated at the ends of initially unimolecular micelles, which subsequently drive the preassemblies to periodically proceed into the hierarchically micellar connection, axial growth, bending, and cyclization processes from nanoscopic assemblies to macroscopic particles. Of particular interest would be systems that acquired the shape control and size adjustment of self-assemblies after termination or reactivation of disulfide reshuffling reaction by regulating external stimuli whenever needed. Such a hierarchical strategy for self-assembled evolution is universally applicable not only for other disulfide-linked dendritic polymers but also for exploitation of biological applications.

\footnotetext{
${ }^{1}$ Beijing National Laboratory for Molecular Sciences, State Key Laboratory of Polymer Physics \& Chemistry, Institute of Chemistry, Chinese Academy of Sciences, Beijing 100190, China. ${ }^{2}$ Pacific Northwest National Laboratory, Richland 99352 WA, USA. ${ }^{3}$ College of Materials Science and Engineering, Zhengzhou University, Zhengzhou, Henan 450001, China. ${ }^{4}$ University of Chinese Academy of Sciences, Beijing 100049, China. Correspondence and requests for materials should be addressed to D.W. (email: dcwu@iccas.ac.cn)
} 
T he "bottom up" or "engineering up" approach from a single molecule to functional architectures has a great significance in understanding biologically spontaneous self-assembly process, which offers an efficient strategy for creating a myriad of well-organized nanostructures in applications of drug delivery, cosmetics, dispersant technology, photonic detector, sensor, and nanoreactor arenas ${ }^{1-12}$. Recently, a great effort has been focused on the dynamic process of solutionassembled nanostructures in highly selective solvents through the elaborate design of the molecular architectures and careful control of the external environments to study the real-time selfassembled evolution. The preparation of shape-shifting and sizegrowing structures is emerging as one of the advanced strategies in organic-based nanoscience and nanotechnology, because most of the smart functionalities of nanocarriers are directly determined by their shapes and sizes, which not only provides detailed information on the structural transformation but also guides the outcome of morphology and functionality in response to outside stimuli. Frequently used methodologies to create temporal evolution of the self-assembly pathway always require precise polymer syntheses, sophisticated architectural design (hydrophobic/ hydrophilic balance, composition, geometry, dimension, surface chemistry, and flexibility ${ }^{13-35}$, fine control of the environmental conditions $(\mathrm{pH}$, temperature, stress, photons, ultrasound, ionic strength, and solvents) and sophisticated optimization of solution assembly kinetic pathways ${ }^{36-45}$. Nevertheless, these methods are still unable to delicately manipulate the self-assembly system and veritably obtain the full-scale solution assemblies due to the difficulty in achieving the precise control of amphiphilic inherent essences at any moment once the self-assembly system is initiated. In other words, regulation of real-time morphology evolution with an instant on/off function at arbitrary time to gain the desired self-assemblies remains a grand challenge. To circumvent this problem, it is greatly desired to design the manipulative reactions and conditions to tailor self-assembly systems with the strict criteria that the self-assembly behaviors should be able to be activated, controllably terminated and interrupted, and reinitiated by the external stimuli whenever needed, because many envisioned applications endeavor to exploit solution assemblies as intelligent nanocontainers for the high encapsulation and controlled release of small molecule cargoes, requiring a detailed understanding of the dynamic processes, long-term stability, and stimulative responsibility of solution assemblies.

Our previous studies exploited a living controlled hydrogel formation method for tailor-made in situ gelling systems ${ }^{46,47}$. Specifically, a star-shaped POSS-(SS-PEG) 8 molecule (Supplementary Fig. 1), containing a disulfide-linked core/shell structure with one polyhedral oligomeric silsesquioxane (POSS) core and eight easy-leaving polyethylene glycol (PEG) shells, was adopted to produce various hybrid hydrogels with customized structures and properties based on a $\mathrm{pH}$-responsive on/off reaction. Supplementary Fig. 2 illustrated the mechanism of $\mathrm{pH}$-triggered thiol-disulfide exchange reaction that could result in the dissociation of PEG shells and linkage of POSS cores. The driving force was shifting of equilibrium toward the stable disulfidelinked products through the release of PEG shells on account of the selective core/shell separations. So, rational control of system $\mathrm{pH}$ and reaction time can tailor the hydrophobic/hydrophilic ratios and topologies, thus manipulating the self-assembled architectures and dimensions in aqueous media. In this case, it is believed that the continuously time-dependent morphology transformation and size evolution with an instant on/off function can be facilely achieved by this switchable method. Of particular interest would be systems that acquired the shape control and size adjustment of self-assemblies after termination or reactivation of the disulfide reshuffling reaction by regulating the system $\mathrm{pH}$.
Herein we propose a living hierarchical self-assembly strategy to manipulation of the morphology and size evolution in situ by tuning the $\mathrm{pH}$ stimuli to activate and/or terminate the thiol-disulfide exchange reaction of the amphiphilic POSS-(SS$\mathrm{PEG})_{8}$ polymer (Fig. 1). By activing the disulfide reshuffling reaction in aqueous solutions, the constructive linkage of POSSembedded domains and removal of PEG segments caused the amphiphiles gather into unimolecular micelles, which acted as the pre-assemblies that further proceeded to the micellar connection, axial growth, bending, and cyclization processes driven by the reactive points at the ends with high-sufficient activity, presenting a periodically hierarchical self-assembly behavior on the all of nanoscales, microscales, and macroscales. Besides, the rigid POSS-embedded backbone and strong aggregation ability can furnish these POSS-containing hybrid polymers with unique selfassembly behaviors ${ }^{48-57}$, thereby generating various nanostructures with a second level of hierarchy. Notably, such a realtime self-assembled evolution could be interrupted at any point of time by neutralization or restarted by rebasification. Using this principle, many sophisticated materials with controlled structures and special properties may have great applications in biomedical platform.

\section{Results}

pH-triggered morphology and size evolution in situ. Effective adjustment of thiol-disulfide exchange reaction kinetics is crucial for acquiring more opportunities to observe the self-assembled evolution. With the aim of reducing the exchange reaction rate and avoiding the gelation, the concentration of POSS-(SS-PEG) polymer was set as $1 \mathrm{mg} / \mathrm{mL}$ in $\mathrm{pH} 12$ solutions. The good hydrophilicity of PEG shells made the amphiphilic polymers disperse well and keep stable after basification for $5 \mathrm{~min}$, forming the unimolecular micelles with the diameter of $\sim 5 \mathrm{~nm}$ (TEM) and $3 \mathrm{~nm}$ by all-atom molecular dynamics (MD) simulation (Supplementary Fig. 3). After addition of catalytic amounts of cysteamine, exchange reaction was immediately activated along with the initiate of PEG chains dissociation and POSS core linkage. With a greater extent of exchange, more PEG segments were released and a higher degree of crosslinking reaction was achieved. Accordingly, the hydrophobic/hydrophilic ratio was continuously increased along with the ongoing variation of dendritic topology. Under this circumstance, the solution assemblies in situ transformed from unimolecular micelles to spherical micelles to cylinders to vesicles to worm-like micelles then to hollow spheres and finally to elliptic nanoparticles (Fig. 2). No doubt that the cleavage and recombination of disulfides played important roles in guiding this self-assembled evolution. For this morphology transition, two types of selfassembly mechanisms may be considered and interpreted. One possibility is the continuously morphological formation, destruction and reconstruction processes due to the variation of molecular topology and amphiphilic ratio. However, the disulfide exchange reaction rate was faster than the self-organization process in alkaline environment. In this case, it was believed that the main driving force of morphology transition should be relied on the hierarchical self-assembly behaviors, by which the thiol-disulfide exchange reaction occurred on the surface or interior of previous pre-assembled subunits. So many intricate morphologies were fabricated with a second level of hierarchy, achieving the periodically one-dimensional axial growth, twodimensional cyclization, and internal crosslinking in the whole evolution process.

Investigation into micelle growth mechanism. In order to trace this dynamic morphology evolution and further reveal the 

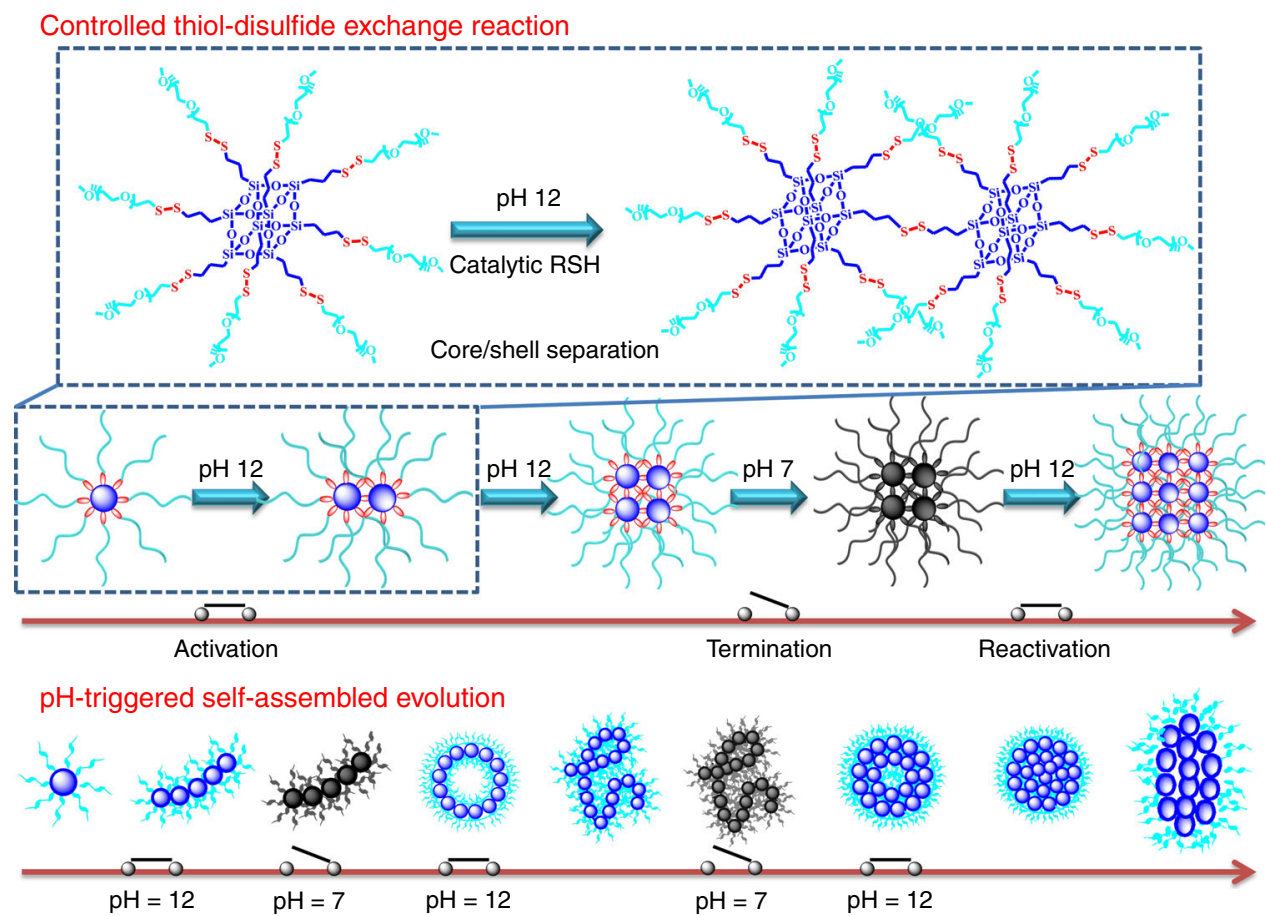

Fig. 1 Controllable strategy for morphology evolution with a pH-switched on/off function. Schematic representation of the living controlled thiol-disulfide exchange reaction and the $\mathrm{pH}$-triggered self-assembled evolution. When the thiol-disulfide exchange reaction is activated in the presence of catalytic thiols in $\mathrm{pH} 12$ solutions, the selective core/shell separation gradually leads to the continuous connections of POSS-embedded cores; in this case, the selfassembled evolution is performed from unimolecular micelles to the elliptic nanoparticles. Once neutralizing the system pH to 7 at any moment, the exchange reaction is deactivated, and thus no further morphology evolution is conducted any more. However, these stable inert sites can be reactivated by basification whenever needed, and the trapped morphology evolution can return back on the previous track

hierarchical self-assembly mechanisms, reduction of the exchange reaction rate was an essential method by adjusting the system $\mathrm{pH}$ to 8. Although the thiols were little activated facilitating the exchange reaction, the structural transformation with a stepwise self-assembly behavior was still performed (Fig. 3). Careful observation found that this dynamic transition process was indeed a kind of second level of hierarchy from the originally molecular-level micelles. Distinct with the traditional hierarchical self-assembly that fully relied on the temperature changes, electrostatic attraction, crystallization, and solvent selectivity, such hierarchical evolution was analogous to the $\mathrm{pH}$-triggered thiol-disulfide exchange reaction that occurred on the surface or interior of the nanoparticles. For simplification purposes, one POSS-SS-(PEG $)_{8}$ unit can be modeled as an isolated core-shell sphere. Based on the sluggish exchange reaction in $\mathrm{pH} 8$ solutions, the unimolecular micelles were slowly connected with each other and formed dimer, trimer, and multimer micellars, just like the structural orientation toward axial growth in one dimension until the formation of short cylindrical structures (Fig. 3a and Supplementary Fig. 4). Moreover, the unique capability of POSS aggregates to form layered crystals is the main driving force in the formation of ellipsoids that seemed like chain-like or lamellar structures parallel to the long axis ${ }^{57}$, so the oligomer micellar connection can gradually assemble into the long and narrow cylinders with low free energy and high priority. AFM and CryoTEM images (Fig. 3b, c) also exhibited the micelle-linked cylinders while DLS results (Fig. 3d) intuitively gave the proof of nanostructural size growth. This referential growth along the axial direction was attributed to the large kinetic barrier and hysteresis effects on the other directions originating from the obstruction of swollen liquid PEG layers, huge hindrance of rigid POSS units and the complex self-assembled pathway in aqueous mediums. Besides, the less coverage of PEG chains at both ends of the micellar aggregates endowed the reactive points at the ends with sufficient activity to induce the following self-assembly into cylinders, presenting a hierarchical stepwise micellar connection towards the axial direction (Fig. 3e).

All-atom simulations of nanostructural axial growth. The limitation of current experimental techniques made obtaining a deep understanding of the mechanism underlying the hierarchical self-assembly difficulty. To address this challenge, we performed MD simulations to intuitively study the axial growth pathways (Supplementary Table 1). When the degree of polymerization was set as 1 and 2, the unimolecular and dimer structures were obtained. As the chain length reached more repeat units, the oligomers exhibited the cylindrical conformations (Fig. 4a). Although the joint sections between the adjacent POSS molecules were flexible, the whole chains could not bend too much due to the steric hindrance effects of side PEG chains. However, this effect also changed the conformation of the side PEG chain, especially for these PEG chains in the medium. Figure $4 \mathrm{~b}$ showed the average root squared gyration radius $\left(R_{\mathrm{g}}\right)$ of middle PEG and side PEG chains in POSS $_{m}-\mathrm{PEG}_{n}$ oligomer. Noticed that the $R_{\mathrm{g}}$ of middle PEG chains was $10 \%$ larger than the side one, indicating the difficulty to approach the center POSS for other active micellar aggregates. So, the lower steric hindrance generated the reactive points at the both ends, driving the oligomers to connect together to form the cylinders along the axis direction with high priority.

Nanostructural bending, cyclization, and radial connection. Due to the flexible linkage and hydrophobic interactions among POSS molecules, these long and dense cylinders slowly bent into the loop to reduce the conformational free energy and finally generated the small vesicles (4-12 h, Fig. 5a). Careful observation 


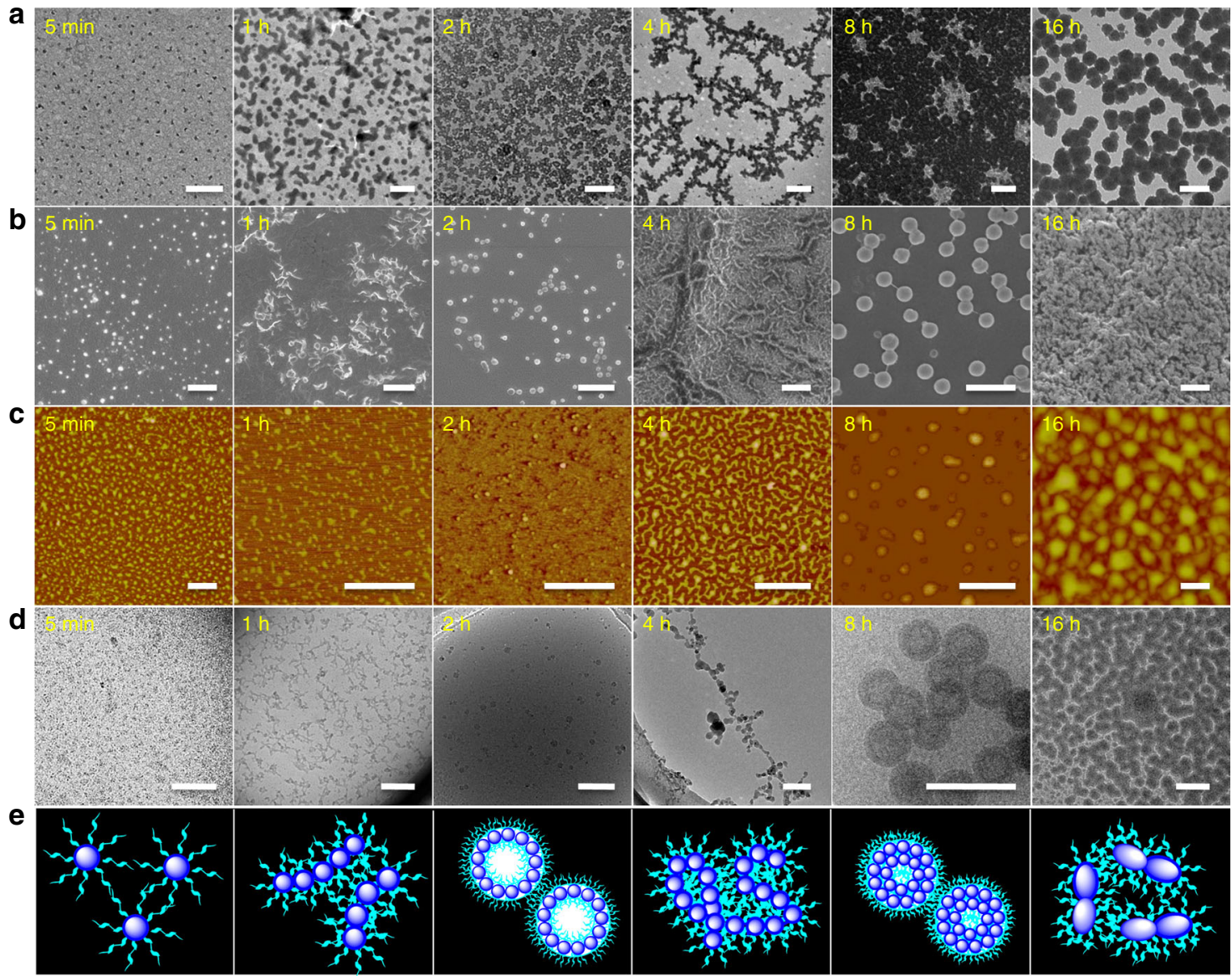

Fig. 2 Multiple measurements of the morphology evolution. After neutralizing the aqueous solution and removing the by-products before dialysis, all the samples are prepared from the neutralized aqueous solution unless otherwise noted. a TEM, b SEM, c AFM, d Cryo-TEM, and e Schematic images showing the self-assembled evolution of POSS-SS-(PEG) 8 with the concentration of $1 \mathrm{mg} / \mathrm{mL}$ in $\mathrm{pH} 12$ aqueous solutions for various times. Scale bars: $0.5 \mu \mathrm{m}$. Along with the initiation of morphology transition in alkaline solutions, multifarious morphologies like uniform micelles, cylinders, vesicles, worm-like micelles, hollow spheres, and elliptic nanoparticles are generated in sequence. The blue sphere represents the hydrophobic POSS core and the cyan chain represents the PEG shell within the self-assemblies

found that the wall thickness of vesicles was approximately equal to the size of one POSS unit (1-2 nm). In other words, it feels like a lot of single POSS molecule standing side by side in a circle to form the wall of vesicle, presenting a monolayer vesicle, which were further testified by multi-techniques approaches (Fig. 5b-d). Supplementary Fig. 5 also provided the evidences to verify the nanoarchitectural bending and cyclization processes on the basis of hierarchical self-assembly behaviors. Besides, the gradual shrinkage of diameter in Fig. 5e indicated the increasing strutural compaction and the decreasing swelling property of these nanoaggregates. On account of the elevated density of disulfide bonds within the narrow mono-vesicles, thiol-disulfide exchange reaction had more possibilities to occur inside and induce the inner cross-connection toward the radial direction, and therefore the interior of vesicles became narrower and compacter until the formation of nanospheres at $16 \mathrm{~h}$. The possibly self-assembled mechanism of nanostructural bending, cyclization, and radial connection process was depicted in Fig. 5f. MD simulations could illustrate the nanostructural bending and radial crosslinking pathways. From above simulation results, the cylinerical micelles presented some flexibility as the chain length is six units on account of the ductile methylene groups in the conjunction. So when the chain length reached tens or hundreds of repeat units, the cylinderical bending and cyclization would be inevitably produced and the aggregates transfered into the irregular patterns like shapeless or distorted vesicles. Because the S-S segment connected to POSS unit with all trans conformation is about $1 \mathrm{~nm}$ within the vesicles, the very short distance between two POSS centers led to the inner neighbor micelles crosslinked with each other, which further made the adjacent micelles closer in return. Therefore, the internal cavity of vesicles was slowly merged into the dense nanospheres, presenting a intramolecular crosslinking pathway.

Periodically hierarchical self-assembly behaviors. Similarly to the unimolecular micelles, these nanospheres were still supposed to possess high superiority on the growth toward the axial orientation to form the longer and denser worm-like nanoparticles (Fig. 6a), which also ascribed to the high activity of the reactive points at the ends of these nanoparticles though they possessed the thick structures and large sizes. When the selfassembled evolution time reached $20 \mathrm{~h}$, these nanospheres were interconnected with each other and transformed into the wormlike micelles. Further evolution, these worm-like micelles grew longer and thicker (Supplementary Fig. 6) and finally fromed the branch-like morphologies (24-32 h). The formation of dendritic aggregates may ascribe to the accumulation or crossover of the long worm-like micelles. Besides, it was inevitable to form the side-crosslinking micelles owing to their large surfaces and complex spatial stereostructures as well as the low amounts of the hydrophilic PEG chains. After $36 \mathrm{~h}$, the formation of compact 


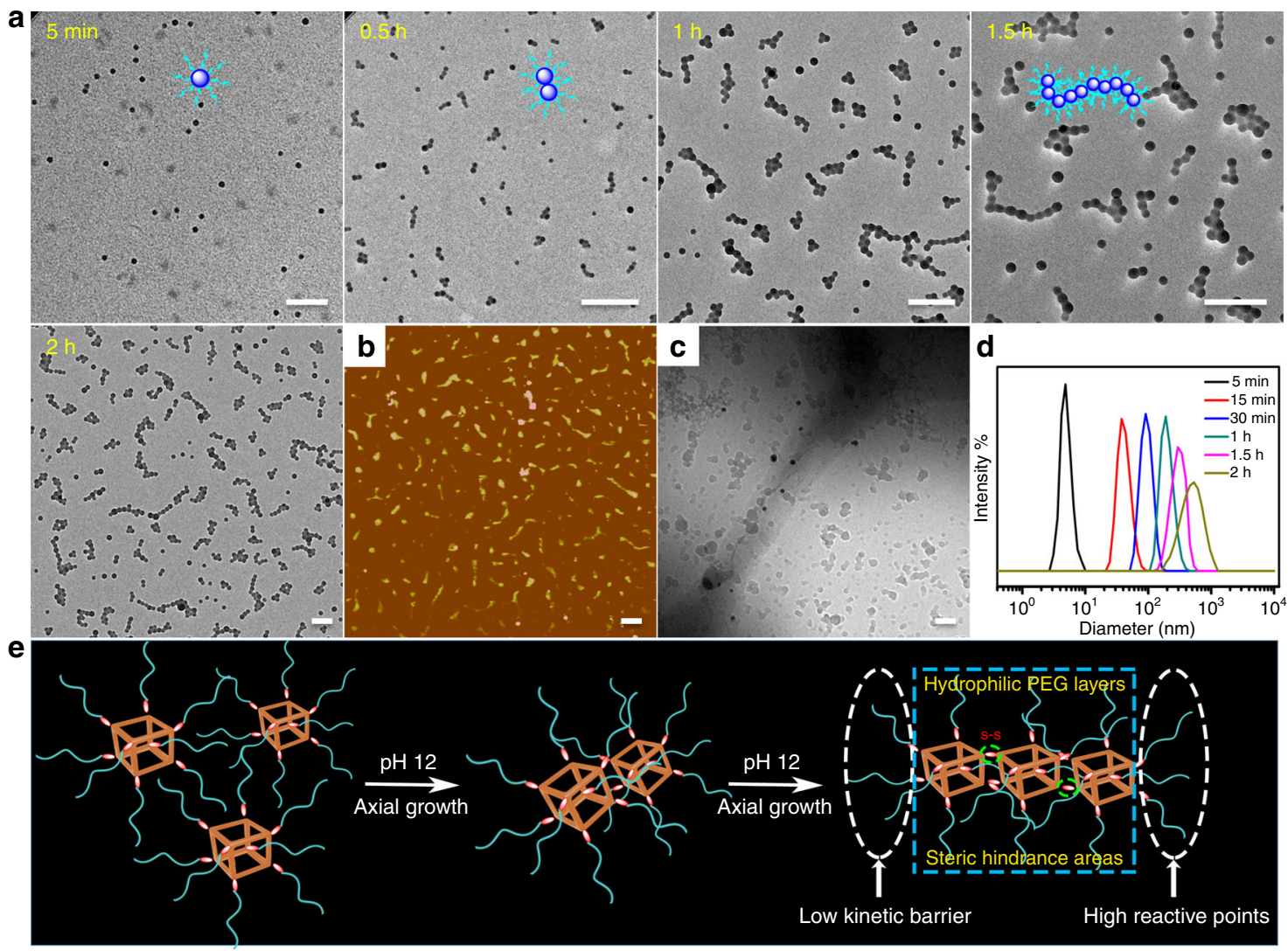

Fig. 3 Mechanism of the hierarchically axial growth of the micelles. After neutralizing the aqueous solution and removing the by-products before dialysis, all the samples are prepared from the neutralized aqueous solution unless otherwise noted. a TEM images showing the self-assembled evolution of POSS-SS-(PEG) 8 with the concentration of $1 \mathrm{mg} / \mathrm{mL}$ in $\mathrm{pH} 8$ solutions for various times. Insets are the correspondingly schematic structures. $\mathbf{b}$ AFM and $\mathbf{c}$ Cryo-TEM images of the short cylindrical formations at $2 \mathrm{~h}$. Scale bars: $50 \mathrm{~nm}$. $\mathbf{d}$ DLS results of the nanoparticles for various times. $\mathbf{e}$ The possible self-assembled mechanism of nanostructural axial growth. In this process, the thiol-disulfide exchange reaction makes the disperse unimolecules quickly aggregate and interlink by the disulfides, thus forming many oligomer-linked cylinders from the pre-micelles with the hierarchical self-assembly behaviors. The low kinetic barrier at both ends, due to the steric hindrance areas from POSS-embedded domains and the swollen layers from stretched PEG shells, furnishes these cylinders with higher reactive points at the micellar ends and drives the subunits to connect together. The orange cube represents the POSS core, the red ellipsoid represents the disulfide linkage and the cyan chain represents the PEG shell

dendritic aggregates exhibited a kind of growth process by the dendritic pathway. SEM, AFM, and Cryo-TEM images (Fig. 6b-d) also presented the longer and denser morphologies with dendritic structures, further verifying the ongoing evolutive tendency of self-assemblies as well as the well-oriented growth pathway at microscopic scales (Fig. 6e).

Afterwards, the cyclization effects induced the evolution further into the hollow spheres ( $~ 50 \mathrm{~nm}$ of thickness, $40 \mathrm{~h})$ for minimal energy, which also proceeded to the radial connection under the command of thermodynamics and dynamics (Fig. 7). The same as the previous stage of morphology and size evolution, the interior of hollow spheres gradually got compacter (44-52 h) as a result of effective exchange reaction within the extremely limited space, thus finally resulting in the formation of solid nanoparticles with the large sizes $(56 \mathrm{~h})$. Besides, the hollow spheres and solid nanoparticles were simultaneously found, convincingly verifying the ongoing evolutive tendency of selfassemblies and revealing the driving forces of shape-shifting by the hierarchical self-assembly behaviors. Repeatedly to former evolution, these large particles tent to accumulate together and further connected with each other to yield the ellipsoidal particles with preferentially axial growth directions (60-72h). Without further morphology evolution, these ellipsoidal particles could maintain their structural stability within a period of time. It was mentioned that the floated fibrous straps were ultimately formed in water after 2 weeks, further verifying their preferentially axial growth behavors, which may reveal the actual hydrogel formation process from nanoscopic solution aggregates to macroscopic hydrogel particles.

For further verification of the hierarchically self-assembled evolution mechanism, in particular morphology changes from spherical micelles to cylindrical micelles and then to vesicles; we integrated the fluorescent markers into self-assembled skeletions to monitor the shape change. On account of the ever-improving hydrophobicity of the assemblies during the thiol-disulfide exchange reaction, aldehyde-functionalized TPE (TPE-CHO) was employed as a hydrophobic aggregation-induced emission (AIE) fluorogen to visually assess the morphology changes. Due to the tight packing of rigid POSS units and TPE segments in hydrophobic sections through strong $\pi-\pi$ stacking, hydrogen bonding, and hydrophobic forces, the intramolecular rotation of TPE groups was restricted extremely in the limited space, thus emitting strong cyan fluorescence. Exactly as the predetermined shape transition mechanisms, these luminous self-assembled nanoparticles transferred from the spherical micelles to narrow cylinders to tortuous vesicles and then to complex micelle-linked cylinders (Fig. 8a), explicitly testifying this hierarchical self-assembly evolution towards the nanostructural axial growth, bending, and cyclization pathways with high priority. 

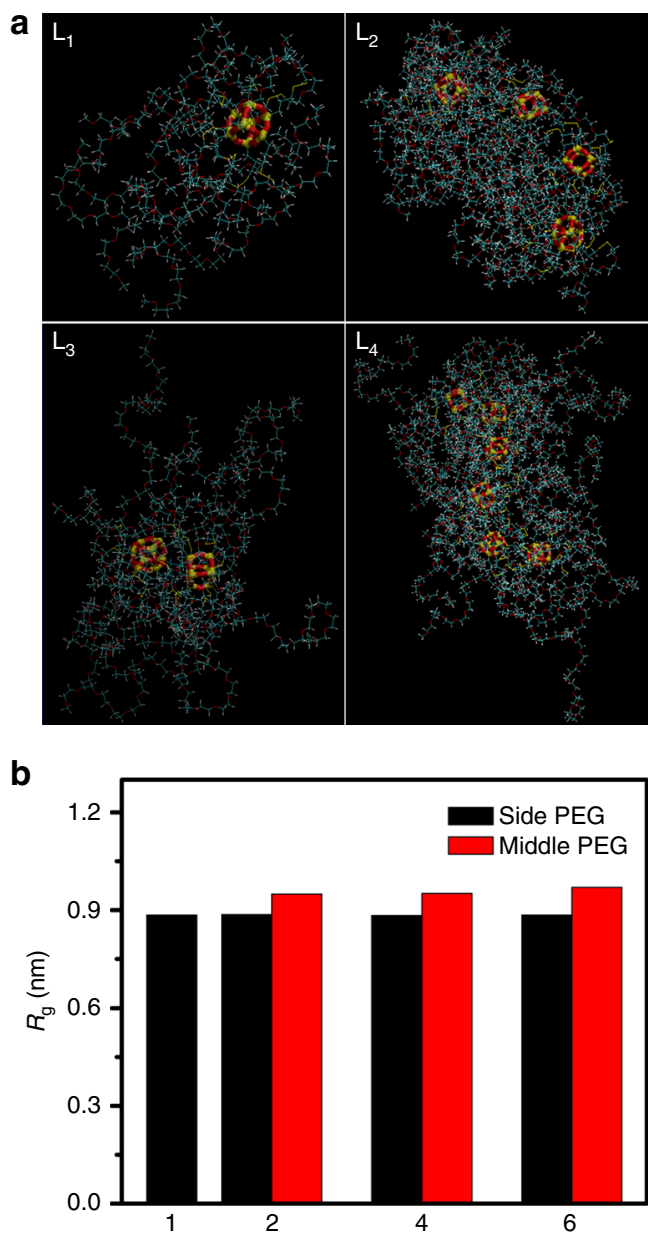

Fig. $4 \mathrm{MD}$ studies of the micellar axial growth mechanism. a The typical conformation of $\mathrm{POSS}_{m}-\mathrm{PEG}_{n}$ oligomer in water with various chain lengths at $\left(L_{1}\right) 1,\left(L_{2}\right) 2,\left(L_{3}\right) 4$, and $\left(L_{4}\right) 6$. Water molecule is not shown for clarity. The inner cube represents the POSS core, wherein the yellow cylinder represents the silicon $(\mathrm{Si})$ atom and the red cylinder represents the oxygen (O) atom. The outer linkage represents the PEG shell, wherein the red cylinder represents the oxygen $(\mathrm{O})$ atom, the yellow cylinder represents the sulfur (S) atom, the wathet blue cylinder represents the carbon (C) atom, and the white cylinder represents the hydrogen $(\mathrm{H})$ atom. $\mathbf{b}$ The average $\mathrm{Rg}$ for PEG chain on the side and middle of POSS molecules (see

Supplementary Table 1 for the detailed simulation parameters)

From the above analysis, we can conclude that this selfassembled evolution was strongly relied on an efficient stepwise strategy for fabricating various nanostructures with a second level of hierarchy (Fig. 8b). By activation of thiol-disulfide exchange in alkaline solutions, the well-defined unimolecular micelles, based on the rigid backbone and special aggregation ability of POSS units, quickly aligned and oriented along the axial growth to form the cylindrical nanostructures. Further elongation and bending, the long cylinders transferred into monolayer vesicles for minimizing the free energy of systems. Afterwards, due to the reduced distance of reactive points and elevated concentrations of disulfides inside the vesicles, the internal crosslinking preferentially occurred like a radial connection to compact the vesicles. In views of this synchronous outer axial growth and inner circumferential crosslinking, these distorted vesicles were transformed into the thickly worm-like nanoparticles. After the following elongation, bending, and cyclization process of chainlike nanostructures, the dense hollow particles were generated by hierarchical self-assembly behaviors. Mentioned that the limited cavity furnished the unreactive points with more possibilities to accelerate the thiol-disulfide exchange reaction, and thus the small hollow particles quickly shrank inward and simultaneously elongated axially for ovalization to form the final ellipsoid particles. Therefore, by means of the $\mathrm{pH}$-triggered thiol-disulfide exchange reaction on the surface or interior of nanostructures, an intriguing morphology and size evolution of self-assemblies was well-conducted under the guidance of hierarchical strategy for circulating axial growth, cyclization, and radial crosslinking process, which was universally applicable at all of nanoscopic, macroscopic, and microscopic scales.

Investigation on exchange reaction and assembly conditions. Comprehensive analysis of this living thiol-disulfide exchange reaction in various conditions is beneficial for acquisition of unusual amphiphilic intermediates and creation of nanoarchitectures. To further study the exchange reaction and assembly condition-controlled morphology and size evolution, monomer concentration, reaction atmosphere, cosolvent effect, and UV switcher were taken into consideration for optimal regulation. Firstly, by reducing the polymeric concentration $(0.1 \mathrm{mg} / \mathrm{mL}$ in $\mathrm{pH}$ 12) to slow down the reaction rate, the similar morphology evolution procedure including spherical micelles, cylinders, vesicles, worm-like micelles, hollow spheres, and elliptic nanoparticles was observed in Supplementary Fig. 7a, which indicated the strong POSS aggregation ability and the corresponding axial growth pathway on the microscales and macroscales were not relied on the concentration of POSS-containing polymers that was consistent with the previous report ${ }^{57}$. In addition to the arrangement of continuously shape-shifting nanostructures in aqueous solutions, we also utilized the mixed solvent of $\mathrm{THF} / \mathrm{H}_{2} \mathrm{O}(v / v=1: 4, \mathrm{pH} 12)$ to simultaneously retard the thiol-disulfide exchange reaction and weaken the POSS aggregation capacity. Thereinto, THF provided the POSS components with sufficient possibilities to gather together, so the cylinders could possess thicker backbones with slower axial growth for a period of time. The size and number increase of micelles could effectively reduce the interfacial area per chain and lower the free energy of system. Nevertheless, the steric hindrance effects of side PEG chains and the symmetrical cage structure of POSS units could still cause the oligomers to connect together with high priority at the axis direction and then undoubtedly bend into the loop to generate hollow spheres (Supplementary Fig. 7b). Without fast shape transition of inner circumferential crosslinking behavior, these hollow spheres were slowly interconnected with each other to form the larger aggregated clusters with concentrated, close-grained and hollow networks, which may be attributed to the severely reducing exchange reaction rate in THF environment although the distances of plentiful POSS centers were narrow within the hollow cavity. This result indirectly demonstrated the existence and significance of circumferential crosslinking process. Moreover, we changed the inert atmosphere $\left(\mathrm{N}_{2}\right)$ and not found obvious difference of periodical transformation process, further proving the facile manipulation of thiol-disulfide exchange reaction and hierarchically nanostructural connection, axial growth, bending, and cyclization pathways (Supplementary Fig. 7c). Besides, on account of the dynamic disulfide bond that could also undergo exchange reaction with radical-mediated mechanism under UV irradiation, we adopted the photochemical method to adjust the reactive sites and regulate the UV-triggered self-assembly behaviors. In contrast to the thiolates-mediated exchange reaction with the driving force of selective core/shell separations, UV irradiation can damage one disulfide to form two freely thiyl radicals that induced the random radical-centered disulfide 


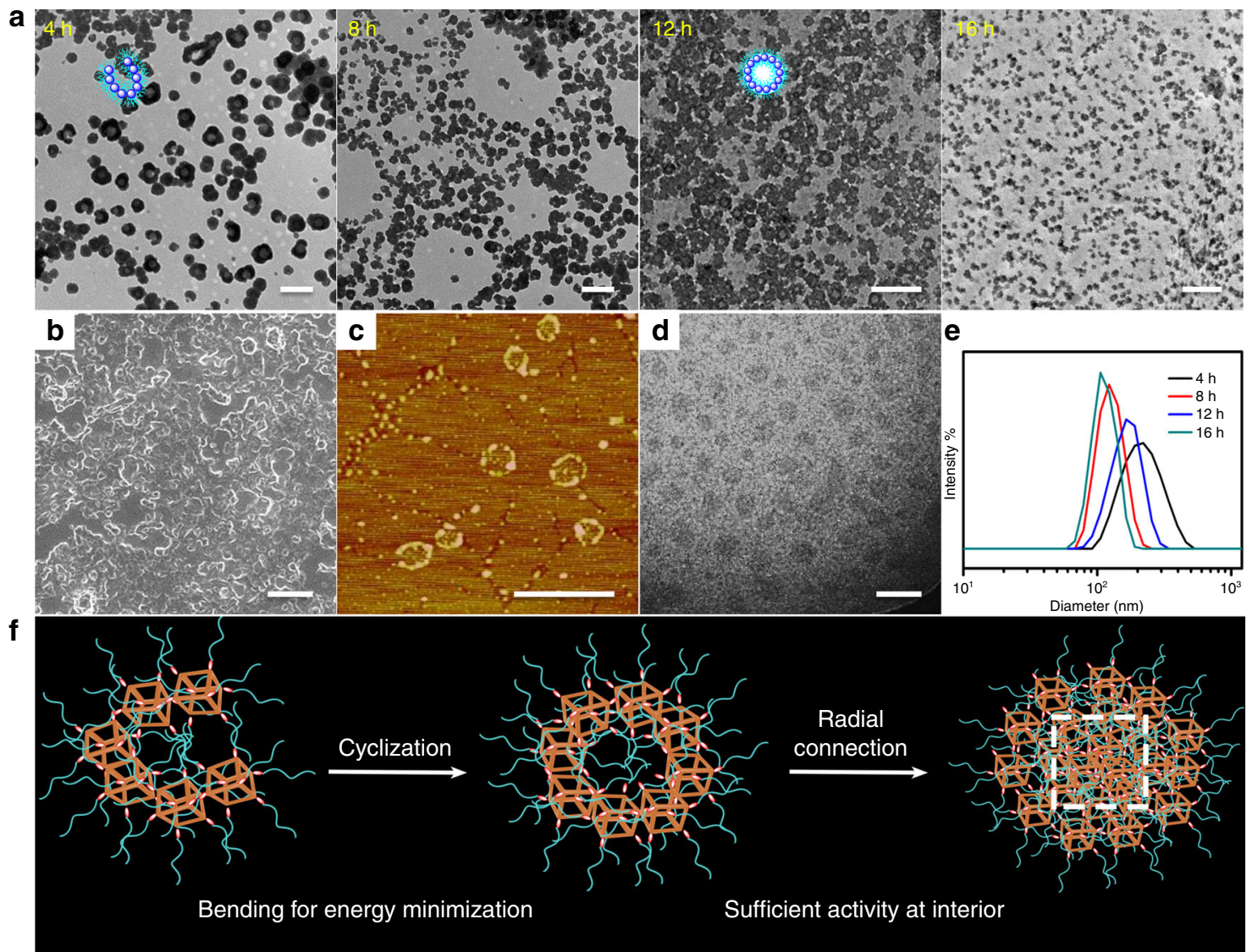

Fig. 5 Mechanism of the hierarchical bending, cyclization, and radial connection of the aggregates. After neutralizing the aqueous solution and removing the by-products before dialysis, all the samples are prepared from the neutralized aqueous solution unless otherwise noted. a TEM images showing the self-assembled evolution of POSS-SS-(PEG) 8 with the concentration of $1 \mathrm{mg} / \mathrm{mL}$ in $\mathrm{pH} 8$ solutions for various times. Insets are the correspondingly schematic structures. b SEM, c AFM, and d Cryo-TEM images of the monolayer vesicular formations at $12 \mathrm{~h}$. Scale bars: $0.5 \mu \mathrm{m}$. e DLS results of the nanoparticles for various times. $\mathbf{f}$ The possible self-assembled mechanism of nanostructural bending, cyclization, and radial connection process. In this process, the longer cylinders can bend into the vesicles for conformational minimal energy. The whole formation process of the vesicles is clearly observed from the TEM images. On account of the limited space at the interior of the vesicles, the number, and density of disulfide bonds is significantly increased so that the possibility of thiol-disulfide exchange reaction is accordingly improved, gradually resulting in the radial connection to form the dense spheres. The orange cube represents the POSS core, the red ellipsoid represents the disulfide linkage and the cyan chain represents the PEG shell

exchange reaction, giving rise to more intricately intermediate structures and multifarious morphologies. As shown in Supplementary Fig. $7 \mathrm{~d}$, the emergence and growth of cylinders were quickly formed under UV irradiation, followed by the generation of a very thick close-grained network as a result of UV-triggered multiple reactive sites and spatiotemporal precision for simultaneous multi-thiol-disulfide exchange reaction, but this random thiol-disulfide exchange mechanism led to the poor-selective core/shell separation and ill-defined morphlogy evolution. More importantly, UV irradiation could destroy the high-density disufides incidentally so that the thicker cylinder-linked networks were dissociated into a mass of shuttle-shaped sheets. Along with the concurrently random thiol-disulfide exchange and degradation reactions, prolonging irradiation time inconceivably resulted in the following square sheets, triangle-shaped plates, rhombus-shaped nanosheets, and silkworm particles, which revealed the great significances of selective core/shell separations on the tremendous contributions of reactive points at the ends with high-sufficient activity. These control experiments reflected that these $\mathrm{pH}$-triggered disulfide reshuffling reaction and the corresponding self-assembly behaviors could be a facilely and efficiently hierarchical self-assembly strategy on guiding morphology and size evolution at nanoscales, microscales, and macroscales.
Dissipative particle dynamics (DPD) simulation results. In fact, thiol-disulfide exchange reaction and self-assembly process occurred simultaneously, which was still a challenge to track in the experiments. Limited by the computational power, it was difficult to study the collective behavior of macromolecules with all-atom model. To overcome this challenge, we performed several coarse-grained simulations to better understand the selfassembly process. Here we employed DPD simulations to study the self-assembly behavior of $\mathrm{POSS}_{m}-\mathrm{PEG}_{n}$ oligomer, which is currently recognized as a viable simulation approach to intuitively study the macromolecular expression and formation pathway for morphology evolution ${ }^{58-63}$. Self-assembled structures can be quantitatively determined by the geometrical packing parameter $P$, where $P=v / a_{\mathrm{o}} l_{\mathrm{c}} \cdot a_{\mathrm{o}}$ is the area of hydrophilic portions while $v$ and $l_{\mathrm{c}}$ are the volume and length of hydrophobic compartments, respectively. With the increase of $P$, the amphiphiles underwent the morphological transition from the unimolecular micelles to dense nanospheres. However, the conventional packing theory cannot interpret the formation of various morphologies in the self-assembly of POSS-(SS-PEG) 8 polymer. As above mentioned, these assemblies were more abundant in contrast to those of traditional amphiphiles, which ascribed to that both the individual surfactant molecules and the assembled structures were dynamic during the self-assembly process. For a better 


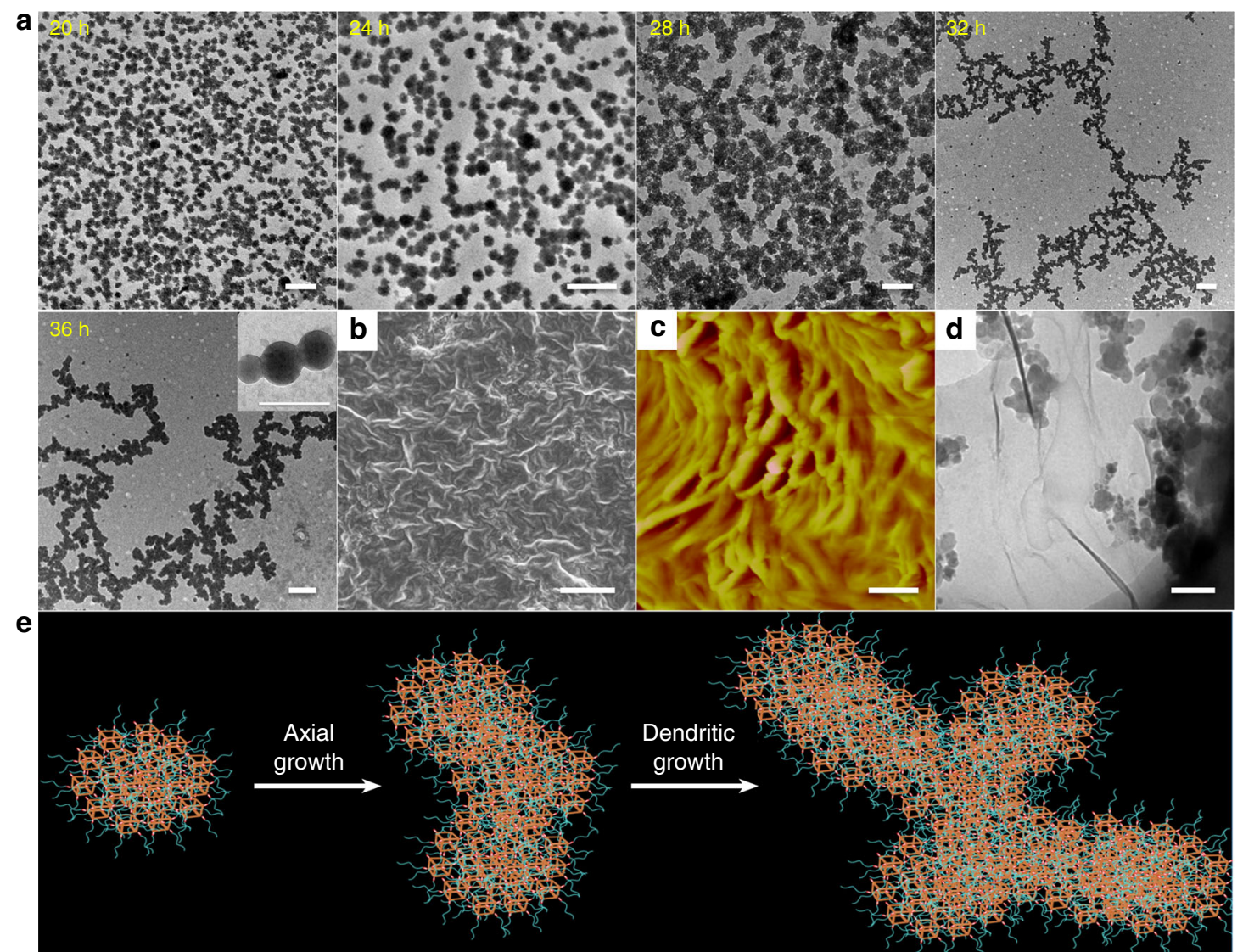

Fig. 6 Hierarchically nanostructural orientation of axial and dendritic growth at microscales and macroscales. After neutralizing the aqueous solution and removing the by-products before dialysis, all the samples are prepared from the neutralized aqueous solution unless otherwise noted. a TEM images showing the self-assembled evolution of POSS-SS-(PEG) 8 with the concentration of $1 \mathrm{mg} / \mathrm{mL}$ in $\mathrm{pH} 8$ solutions for various times. The inset is the high magnification image of worm-like formations at $36 \mathrm{~h}$, intuitively proved the interconnect arrangement of self-assemblies. b SEM, c AFM, and d Cryo-TEM images of the worm-like formations at $36 \mathrm{~h}$. Scale bars: $0.5 \mu \mathrm{m}$. e The possible self-assembled mechanism of nanostructural growth with axial and dendritic directions. In this process, the large scale nanoparticles are still grown toward the axial orientation roughly at first, but finally formed the dendritic wormlike micelles with large size. The complex architectures, giant surface areas and reduced hydrophilic layers of the spheres provide more chances for the side-crosslinking to form the dendritic conformations. The orange cube represents the POSS core, the red ellipsoid represents the disulfide linkage and the cyan chain represents the PEG shell

understanding of self-assembly mechanism and intuitive observation of morphology transition, we performed DPD simulations using CG models in Supplementary Fig. 8. The morphology transferred from unimolecular to spherical micelles as the hydrophobic interaction increased between the POSS molecules (Fig. 9a-d). As the hydrophobic interaction improved, which was equal to the larger size of POSS-linked cores in the experiments, the cylindrical and vesicular structures appeared (Fig. 9e-g). This could be attributed to that the restriction of rigid POSSembedded cores had a significant influence on the conformation of flexible chains, e.g., the stretching and compacting of PEG chains that yielded the diagram of varied assemblies. When the hydrophobic interaction between adjacent POSS-domains cores and water was further increased, the long worm-like micelles and hollow spheres occurred in Fig. 9h-l. Additionally, the topology constraint could also influence the shape transformation of aggregates. For example, the enough exposed surface area of POSS core was required if acquisition of other morphologies. As shown in Fig. 9m-o, dense nanospheres were obtained when the number of peripheral PEG chains reduced to a quarter. Further evolution, the elipsoid nanoparticles were finally formed in Fig. 9p. These DPD simulations further testified the process of morphology evolution in theory.
pH-Switched morphology evolution with an on/off function. Once the thiol-disulfide exchange was interrupted at any moment by neutralizing the system $\mathrm{pH}$, the thiolates of assemblies were deactivated to thiols, thus no further exchange reaction was conducted any more as well as the termination of hierarchical self-assembly behaviors. However, these stable inert thiols and trapped morphology evolution could be reactivated whenever needed. For instance, when the polymer solution was basified at $\mathrm{pH} 12$ (Fig. 10), the short cylinders were formed around $30 \mathrm{~min}$ later and further transferred into vesicles after $2 \mathrm{~h}$. By neutralizing solutions at $\mathrm{pH} 7$, this transition could be interrupted and the cylindrical micelles were kept stable for a long time. However, after rebasification of system $\mathrm{pH}$ to 12 , the morphology transition was restarted to evolve into the vesicles. Similarly, the stable worm-like micelles could also be yielded by interruption of morphology evolution in aqueous solutions. These neutralized self-assemblies had a good long-term stability and basically maintained their shapes and sizes at least 7 days in mild aqueous environments. These results indicated a simple method to differentiate and gain the complex and intermediate morphologies. Therefore, the conceivable self-assemblies with customized structures and properties can be easily tailored only by the control of system $\mathrm{pH}$ within a predetermined time. 


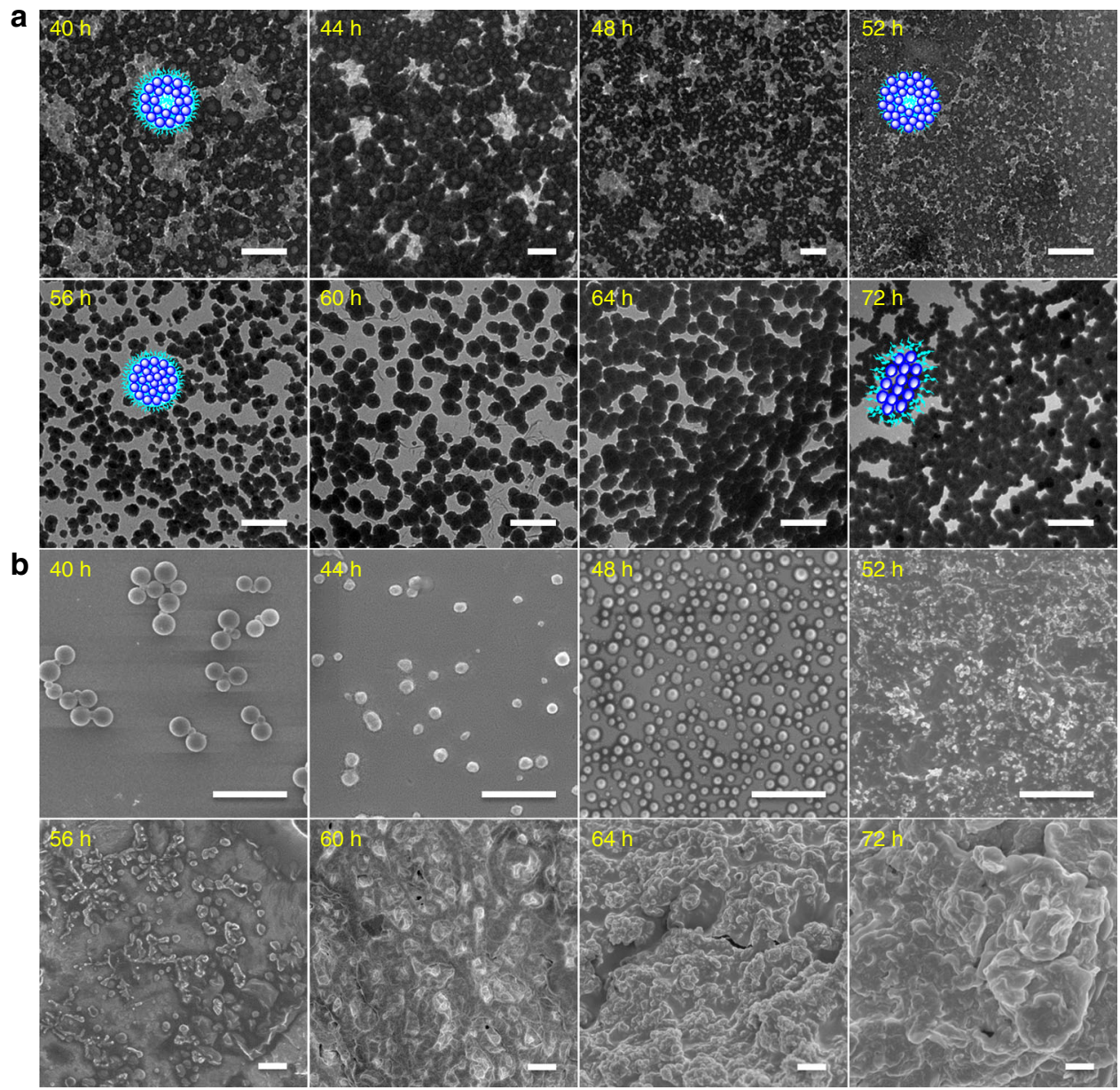

Fig. 7 Hierarchically nanostructural bending, cyclization, and radial connection at microscales and macroscales. After neutralizing the aqueous solution and removing the by-products before dialysis, all the samples are prepared from the neutralized aqueous solution unless otherwise noted. a TEM and $\mathbf{b}$ SEM images showing self-assembled evolution of POSS-SS-(PEG) 8 with the concentration of $1 \mathrm{mg} / \mathrm{mL}$ in $\mathrm{pH} 8$ aqueous solutions for various times. Insets are the correspondingly schematic structures. Scale bars: $0.5 \mu \mathrm{m}$. The formation of hollow spheres verifies the nanostructural bending and cyclization mechanism. The reduced size of vesicle cavity demonstrates the inner circumferential crosslinking process, and the following spherical and elliptic aggregates confirm the periodically axial growth pathway even on the microscales and macroscales, which indicates the achievement of hierarchical self-assembly strategy for the guiding the morphology and size evolution from the nanoscopic solution aggregates to the microscopic and macroscopic hydrogel particles

Importance of uniform core on the hierarchical behaviors. According to the aforementioned analysis, we conjecture that this living hierarchically assembled strategy for self-assembled evolution should be recognized as a versatile method because it may also be suitable for other materials with similar disulfide-linked dendritic architectures. To validate this proposal, we produced a disulfide-linked hyperbranched poly(BAC2-AEPZ1)-PEG polymer with an irregular core by Michael addition polymerization (Supplementary Fig. 9) ${ }^{46}$. As seen in Supplementary Fig. 10, a scattered anisotropic variation of assemblies, mainly driven by the hydrophobic interactions, were conducted in alkaline solutions. The simple axial growth was observed in the first stage on account of steric hindrance areas from hydrophobic domains and the swollen layers from the stretched PEG shells at the middle of micelles. However, as a result of random linkages by the newlyformed disulfides among the nanoparticles, hierarchical selfassembly failed to perform the cylindrical bending and circumferential crosslinking behaviors, which indicated the importance of the uniformity and rigidity of dendritic core. To further verify this principle, we conducted another control experiment using a well-defined but asymmetric cyclodextrin $(\beta-\mathrm{CD})$ core to obtain the $\beta$-CD-(SS-PEG) $3-4$ polymer via an acylation reaction
(Supplementary Fig. 11), which self-assembled into unimolecular micelles at the original stage and further transferred into the worm-like micelles in $\mathrm{pH} 12$ solutions. On account of the uniform size and highly crosslinking CD cores, these aggregates performed the unequivocal step-change shifts in cylindrical micelle length and population during the process of thiol-disulfide exchange reaction. The stacking $\beta$-CD molecules played an important role in the micellar dimensional growth. As shown in Supplementary Fig. 12, the sizes increased from unimolecule scale to nanoscale to micrometer scale in alkaline solutions. However, the cyclization and radial crosslinking process were not observed due to the asymmetrical cone, ring tensions, and huge steric hindrance of the cyclodextrin, which further suggested the significance of symmetry on the sequential morphlogy transition. Nevertheless, the living hierarchical selfassembly strategy for size evolution was indeed effective and universal, because once neutralizing the solution, the size growth was interrupted and fixed with a long-term stability. Therefore, different types of polymeric assembly evolution with an on/off function can be easily customized via design of disulfide-linked architectures with uniform and nondeformable cores by means of the living thiol-disulfide exchange reaction. 


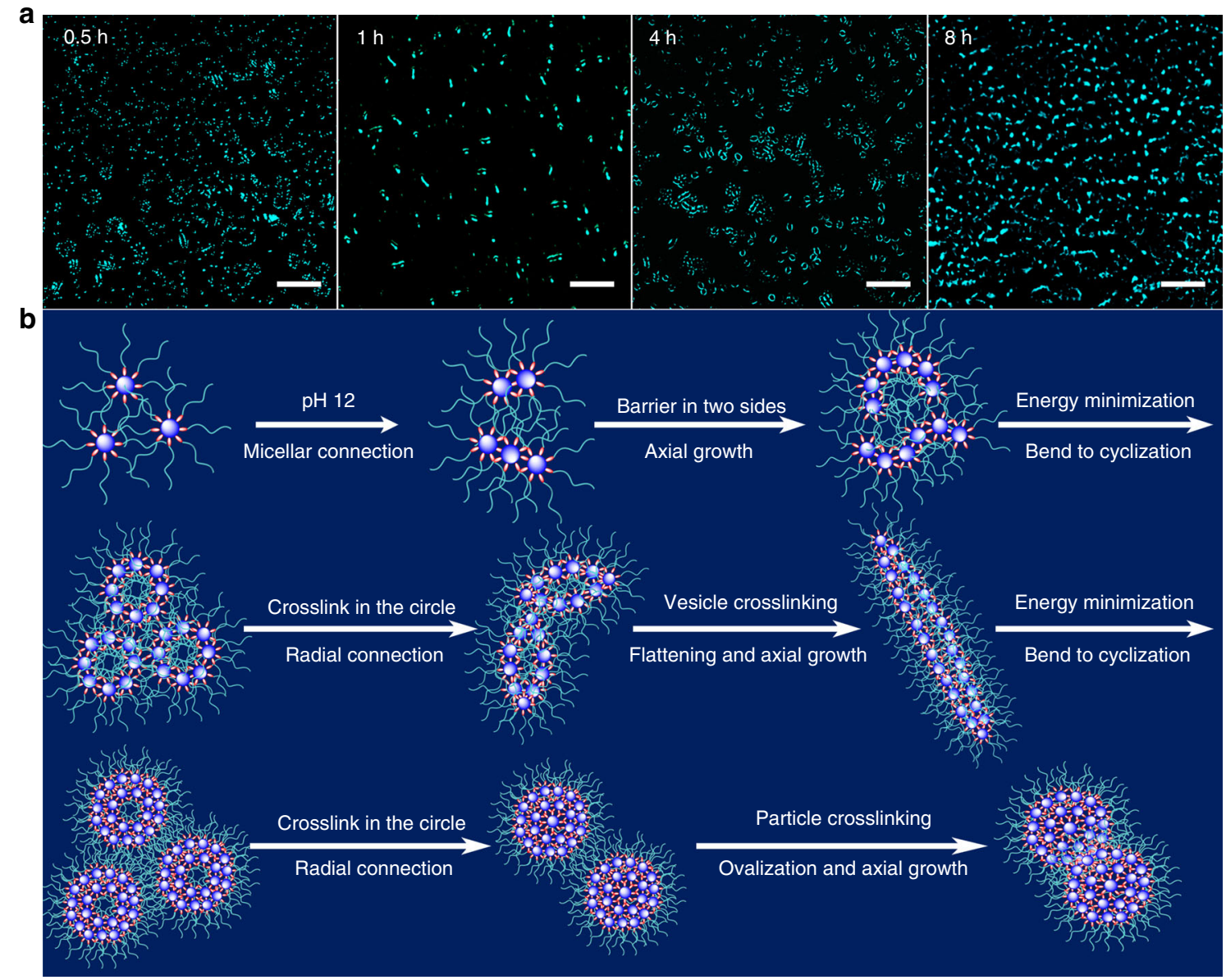

Fig. 8 Hierarchical self-assembly strategy for morphology evolution within the full-scales. a Confocal laser scanning microscopy (CLSM) images of the POSS-SS-(PEG) @ @TPE-CHO aggregates in pH 12 THF $/ \mathrm{H}_{2} \mathrm{O}$ mixtures for various times. Scale bars are $2 \mu \mathrm{m}$. On account of the cage structure of POSS units and propeller shape of TPE-CHO molecules, the POSS-SS-(PEG) 8 @TPE-CHO aggregates could present a little difference of morphology evolution compared to the pure POSS-SS-(PEG) 8 polymer after activation of thiol-disulfide exchange reaction. Nevertheless, the in situ morphology change from micelles to cylinders and then to vesicles was clearly monitored using the fluorescent marker with the prolongation of self-assembly time, intuitively demonstrating the hierarchical morphology evolution process. b Schematic illustration of the whole self-assembled evolution process of the POSS-SS$(P E G)_{8}$ polymer in aqueous solutions, including the possible formation mechanisms of these typical morphologies, such as unimolecular micelles, cylinders, vesicles, worm-like micelles, hollow spheres, dense and elliptic nanoparticles. The blue sphere represents the POSS core, the red ellipsoid represents the disulfide linkage and the cyan chain represents the PEG shell within the self-assemblies

Potential application for drug delivery systems. High loading efficiency and stimuli-responsive property are two pivotal factors for creation of robust nanocarriers. In views of the increased hydrophobicity/hydrophilicity ratio, varied molecular topology and elevated number of disulfide linkages during the morphology transition process, this living self-assembly system was of great significance on the fabrication of redox-responsive drug delivery systems. The encapsulation ability was firstly quantitatively estimated using fluorescent TPE probes in $\mathrm{pH} 12$ solutions. When the AIE-active TPE-CHO molecule was added into the $\mathrm{THF} / \mathrm{H}_{2} \mathrm{O}(v / v=1: 4)$ mixtures of POSS-(SS-PEG) 8 polymer, the activation and process of morphology evolution led to the gradual encapsulation of more and more hydrophobic TPE molecules into the assemblies along with the growth of fluorescent intensity (Supplementary Fig. 13). After incubation for 24 $\mathrm{h}$, the hydrophobicity of aggregates was intensely enhanced so that the precipitates occurred and gradually increased at the bottom and the luminescence of upper clear solution was substantially weakened corresponding to the sharp decline of fluorescent intensity. After sufficient incubation time for 6 days, the separated white precipitates emitted strong cyan fluorescent light while the supernatant liquid was nearly non-emissive, suggesting the complete encapsulation of TPE-CHO into polymeric assemblies.

As for the encapsulation of antitumor drugs, taking account of the incompatible alkaline environment for protein and live cells, we changed into $\mathrm{pH} 7.4$ to in situ trigger the thiol-disulfide exchange reaction though it owned a very low reaction rate. Nevertheless, the drug-loading efficiency could be still up to $70 \%$ (Supplementary Fig. 14a) along with the increase of hydrophobic/ hydrophilic ratio and variation of molecular topology. Similarly to the $\mathrm{pH}$-switched self-assembly behaviors, the drug-loaded efficiency also possessed a great correlation with thiol-disulfide exchange reaction in various $\mathrm{pH}$ conditions, which could be facilely activated, controllably terminated and interrupted, and reinitiated by the external stimuli (Supplementary Fig. 14b).Then we filtered out two representative DOX-loaded micelles to estimate the intracellular drug delivery after comprehensive considerations of particle size, DTT-triggered release behavior, cell viability, and antitumor activity toward MCF-7 cells (Supplementary Figs. 14c, d and 15a, b). Confocal laser scanning microscopy exhibited the cellular uptake and intracellular drug 


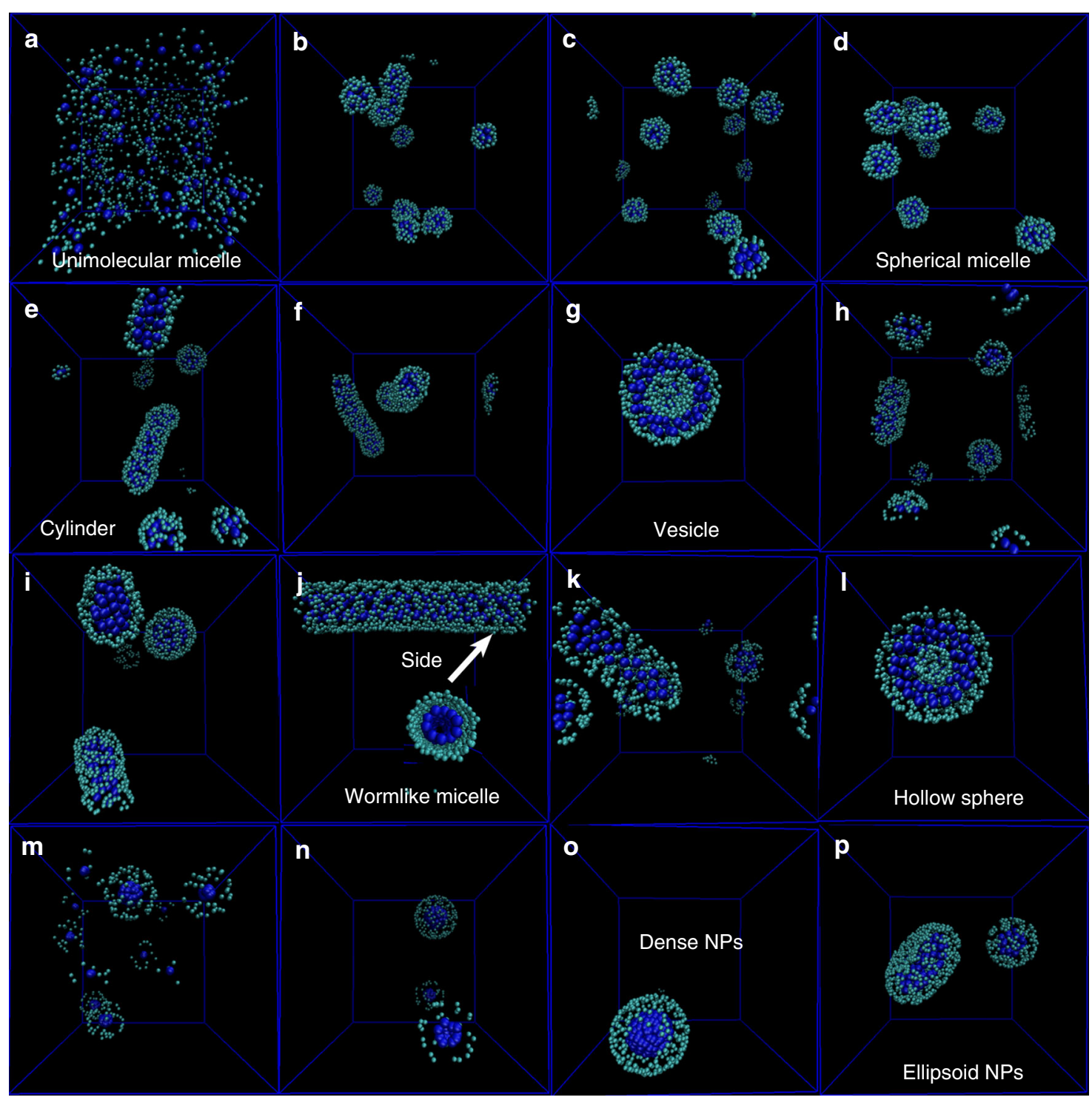

Fig. 9 DPD simulations showing the formation pathway and morphology evolution of self-assemblies. a Unimolecular structure, b-d spherical structures, e cylindrical structures, $\mathbf{f}$ intermediate structures from cylinders to vesicles, $\mathbf{g}$ vesicular structures, $\mathbf{h}$, $\mathbf{i}$ intermediate structures from vesicles to worm-like micelles, $\mathbf{j}$ long worm-like structures, $\mathbf{k}$ curved worm-like structures, I hollow spherical structures, $\mathbf{m}-\mathbf{0}$ intermediate structures of dense nanoparticles, and $\mathbf{p}$ ellipsoid nanoparticle structures. The water beads are omitted for clarity (see Methods section for the detailed simulation parameters)

release behaviors of DOX-loaded aggregates (Supplementary Fig. $15 \mathrm{c}$ ). The enhanced fluorescent intensity revealed the rapid internalization of drug carriers and efficient release of antitumor DOX drugs into the nucleus. Accordingly, the above unique advantages including high drug-loading efficiency, smart redoxresponsibility, and good biocompatibility indicated the great potential biological applications in drug delivery system.

\section{Discussion}

In summary, we demonstrated a tunable manipulation of solution assemblies evolution in situ based on the living hierarchical selfassembly of amphiphilic POSS-(SS-PEG) 8 polymer. Careful tailor of the $\mathrm{pH}$-triggered thiol-disulfide exchange reaction and selfassembled conditions could make the reactive points at the ends of these nanoparticles with high activity, thus presenting the intriguing morphology and size evolutions like periodically micellar connection, axial growth, bending and cyclization processes from the initially unimolecular pre-assemblies on the stepwisely self-assembly behaviors, by which the multifarious morphologies like spherical micelles, cylinders, vesicles, worm-like micelles, hollow spheres, and elliptic nanoparticles as well as the gradual size growths were formed in sequence. Such controllable strategy to guide the hierarchical self-assembled evolution was applicable from the nanoscopic assemblies to microscopic and macroscopic aggregates. Notably, this hierarchical selfassembled evolution could be interrupted and reactivated at any moment only by removal of the external stimuli, and thus the desirable morphology and size was facilely gained with good stability, which provided unique advantages and more opportunities to fabricate the intelligent drug carriers with high loading efficiency and stimuli-responsive property. This hierarchical concept to program the dynamic morphology and size evolution is also universal for other disulfides-linked dendritic polymers with well-structured cores, which will provide useful guidance for achieving more well-worth exploring self-assembly mechanism and artificial superstructures with defined shapes and desired functions. 

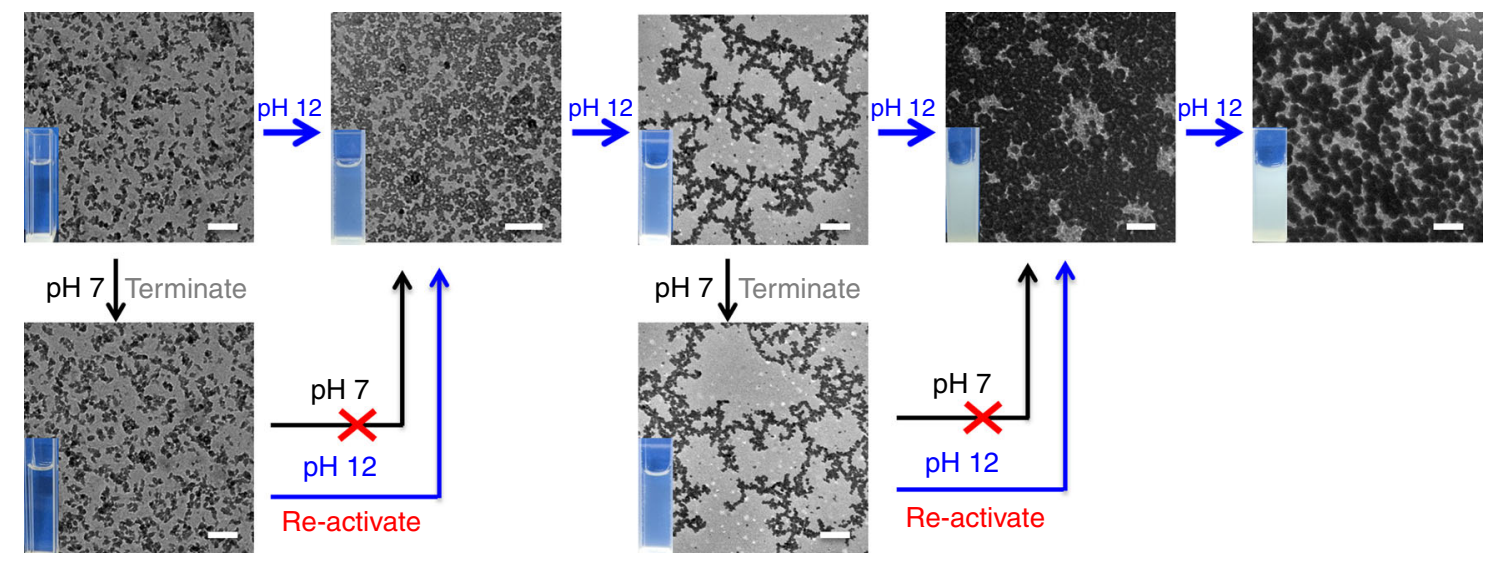

Fig. $10 \mathrm{pH}$-Switched morphology evolution. TEM images showing in situ morphology evolution with termination and reactivation function under the control of system pH. Scale bars: $0.5 \mu \mathrm{m}$. During the whole morphology transition process, once the thiol-disulfide exchange reaction is interrupted by neutralization at any time, the self-assemblies, including cylinders, vesicles, worm-like micelles, hollow spheres, etc. at that moment are fixed and kept stable for more than 7 days, but this morphology and size evolution is easily restarted and returned back on its right track by reactivation of the exchange reaction at $\mathrm{pH} 12$ solutions. This whole self-assembled evolution process can be easily activated, controllably terminated and interrupted, and reinitiated by the external $\mathrm{pH}$ stimuli whenever needed. The inset shows that the assembled solution in the quartz plate is changed from transparency to turbidity

\section{Methods}

Materials and synthesis. (3-Mercaptopropyl) trimethoxysilane (97\%, J\&K), 2,2'-dithiodipyridine (98\%, Energy Chemical), poly(ethylene glycol) methyl ether (PEG-OH, $M_{\mathrm{n}}=750 \mathrm{~g} / \mathrm{mol}, 98 \%$, Energy Chemical), thionyl chloride (97\%, J\&K), 2-mercaptoethylamine hydrochloride (MCA, 99\%, Energy Chemical), 1-(2-aminoethyl)piperazine (AEPZ, 97\%, Aldrich), diisopropanolamine (DIAD, 98\%, Energy Chemical), phthalimide (97\%, Energy Chemical), triphenylphosphine (99\%, Energy Chemical), hydrazine hydrate (95\%, J\&K), triethylamine (TEA, $99 \%$, Beijing Chemical Works), succinic anhydride (99\%, J\&K), $N, N^{\prime}$-bis(acryloyl) cystamine (BAC, $98 \%$, Fluka), $\beta$-CD $\left(>95 \%\right.$, TCI) was dried at $70^{\circ} \mathrm{C}$ under vacuum for $24 \mathrm{~h}$ before use. Cell counting kit- 8 was purchased from Beyotime Biotechnology. DMEM (Corning) with $4.5 \mathrm{~g} / \mathrm{L}$ glucose, L-glutamine, without sodium pyruvate was purchased from Beijing BioDee Biotechnology Co. Ltd. MCF-7 cell lines were purchased from the American type Culture Collection (ATCC, Rockville, MD). Other reagents were commercially obtained from Beijing Chem. Co. and used without further purification.

Synthesis of the PEG-NH $\mathbf{N}_{2}$ polymer. A solution of DIAD $(50 \mathrm{mmol}, 10.7 \mathrm{~g})$ in $20 \mathrm{~mL}$ of DCM was added dropwise to a solution of PEG $\left(M_{\mathrm{n}}=750 \mathrm{~g} / \mathrm{mol}\right.$, $25 \mathrm{mmol}, 18.75 \mathrm{~g}$ ), phthalimide ( $50 \mathrm{mmol}, 7.43 \mathrm{~g}$ ), and triphenylphosphine $(50 \mathrm{mmol}, 13.25 \mathrm{~g})$ in $70 \mathrm{~mL}$ of DCM at room temperature. The solution was stirred at room temperature for 7 days. The solution was removed under reduced pressure. The product was then dissolved in water and removed by filtration and the aqueous solution was washed with diethyl ether. Water was removed under reduced pressure and the oily product of PEG-phthalimido was dried at $40^{\circ} \mathrm{C}$ under high vacuum with $99 \%$ yield. ${ }^{1} \mathrm{H} \mathrm{NMR}\left(\mathrm{CDCl}_{3}, 400 \mathrm{MHz}, \mathrm{ppm}\right): \delta 3.38$ $(\mathrm{s}, 3 \mathrm{H}), 3.73(\mathrm{t}, 2 \mathrm{H}), 3.83(\mathrm{t}, 2 \mathrm{H}), 7.84(\mathrm{~m}, 4 \mathrm{H}) .{ }^{13} \mathrm{C} \mathrm{NMR}\left(\mathrm{CDCl}_{3}, 100 \mathrm{MHz}\right.$, ppm): $\delta 40.6,59.2,65.3,70.4,71.6,123.7,132.2,168.1$.

PEG-phthalimido $(25 \mathrm{mmol}$ ) in $100 \mathrm{~mL}$ of ethanol were treated with $500 \mathrm{mmol}$ of aqueous solutions of hydrazine hydrate under reflux overnight. Concentrated hydrochloric acid was added to the cold solution up to $\mathrm{pH} 2$ and the precipitate was removed by filtration. After evaporation of ethanol, a small amount of water was added to the extracts and aqueous sodium hydroxide was added up to $\mathrm{pH} 10$. The polymers were extracted with DCM for several times. The solution was dried over $\mathrm{MgSO}_{4}$ and after evaporation to obtain the oily PEG-NH $\mathrm{N}_{2}$. Water dilution followed by washing with diethyl ether, and evaporation of water were necessary to fully purify the PEG-NH 2 at $40{ }^{\circ} \mathrm{C}$ under high vacuum with $95 \%$ yield. ${ }^{1} \mathrm{H}$ NMR $\left(\mathrm{CDCl}_{3}, 400 \mathrm{MHz}, \mathrm{ppm}\right): \delta 3.11(\mathrm{t}, 2 \mathrm{H}), 3.38(\mathrm{~s}, 3 \mathrm{H}), 3.67(\mathrm{t}, 2 \mathrm{H}) .{ }^{13} \mathrm{C} \mathrm{NMR}$ $\left(\mathrm{CDCl}_{3}, 100 \mathrm{MHz}, \mathrm{ppm}\right): \delta 41.6,59.3,70.1,70.4,71.6,73.1$.

Synthesis of the PEG-SH polymer. PEG-OH $\left(M_{\mathrm{n}}=750 \mathrm{~g} / \mathrm{mol}, 7.5 \mathrm{~g}, 10 \mathrm{mmol}\right)$ and triethylamine $(7 \mathrm{~mL}, 50 \mathrm{mmol})$ were dissolved in $100 \mathrm{~mL}$ of anhydrous DCM and cooled down to $0{ }^{\circ} \mathrm{C}$ for $15 \mathrm{~min}$. Methanesulfonyl chloride $(4 \mathrm{~mL}, 50 \mathrm{mmol}$ ) was then added to the mixture. After stirring for $5 \mathrm{~h}$ at room temperature, the mixture was quenched with $150 \mathrm{~mL}$ of water, and extracted with $50 \mathrm{~mL}$ of DCM for several times. Then the combined organic layers were washed with water and dried over $\mathrm{MgSO}_{4}$ to obtain the yellow product of $\mathrm{PEG}-\mathrm{SO}_{3} \mathrm{CH}_{3}$ with $99 \%$ yield. ${ }^{1} \mathrm{H} \mathrm{NMR}\left(\mathrm{CDCl}_{3}, 400 \mathrm{MHz}, \mathrm{ppm}\right): \delta 3.15(\mathrm{~s}, 3 \mathrm{H}), 3.38(\mathrm{~s}, 3 \mathrm{H}), 3.71(\mathrm{t}, 2 \mathrm{H})$.

${ }^{13} \mathrm{C} \mathrm{NMR}\left(\mathrm{CDCl}_{3}, 100 \mathrm{MHz}, \mathrm{ppm}\right): \delta 38.2,59.2,67.8,69.2,70.4,72.1$.

As-synthesized PEG-SO $\mathrm{SO}_{3} \mathrm{CH}_{3}(8.2 \mathrm{~g}, 10 \mathrm{mmol})$ and potassium thioacetate (3.6 g, $25 \mathrm{mmol})$ were dissolved in $100 \mathrm{~mL}$ of anhydrous THF under a nitrogen atmosphere. The reaction mixture was degassed three times with $\mathrm{N}_{2}$ and then heated to $60{ }^{\circ} \mathrm{C}$. After stirring for $12 \mathrm{~h}$, the mixture was quenched by water and extracted with DCM. The DCM layer was collected, and residual water was removed with $\mathrm{MgSO}_{4}$ to obtain the yellow product of PEG-SCOCH 3 with $89 \%$ yield. ${ }^{1} \mathrm{H} \mathrm{NMR}\left(\mathrm{CDCl}_{3}, 400 \mathrm{MHz}, \mathrm{ppm}\right): \delta 2.31(\mathrm{~s}, 3 \mathrm{H}), \delta 3.01(\mathrm{t}, 2 \mathrm{H}), 3.38$ $(\mathrm{s}, 3 \mathrm{H}), 3.71(\mathrm{t}, 2 \mathrm{H}), 4.06(\mathrm{t}, 2 \mathrm{H}) .{ }^{13} \mathrm{C} \mathrm{NMR}\left(\mathrm{CDCl}_{3}, 100 \mathrm{MHz}, \mathrm{ppm}\right): \delta 30.5,34.5$, 59.3, 70.4, 71.8,195.0.

As-synthesized PEG-SCOCH $3(6.9 \mathrm{~g}, 8.4 \mathrm{mmol})$ was dissolved in methanol under a $\mathrm{N}_{2}$ atmosphere and degassed three times. Concentrated hydrochloric acid $(0.8 \mathrm{~mL})$ was added to the solution up to $\mathrm{pH} 2$. The mixture was refluxed at $80^{\circ} \mathrm{C}$ for $24 \mathrm{~h}$, quenched with water, and then extracted with DCM. The DCM layer was collected, and residual water was removed by $\mathrm{MgSO}_{4}$ to obtain the final white product of PEG-SH with $82 \%$ yield. ${ }^{1} \mathrm{H}$ NMR $\left(\mathrm{CDCl}_{3}, 400 \mathrm{MHz}, \mathrm{ppm}\right): \delta 1.56$ $(\mathrm{s}, 3 \mathrm{H}), \delta 2.69(\mathrm{t}, 2 \mathrm{H}), 3.38(\mathrm{~s}, 3 \mathrm{H}), 3.81(\mathrm{t}, 2 \mathrm{H}) .{ }^{13} \mathrm{C} \mathrm{NMR}\left(\mathrm{CDCl}_{3}, 100 \mathrm{MHz}\right.$, ppm): $\delta$ 24.1, 59.1, 70.1, 70.4, 71.8, 72.9.

Synthesis of the star-shaped POSS-(SS-PEG) $\mathbf{8}$ polymer. Octa (3-mercaptopropylsilsesquioxane), POSS-(SH) $)_{8}$, was synthesized as follows: $50 \mathrm{~mL}$ of methanol, $10 \mathrm{~mL}$ of acetonitrile, and $8 \mathrm{~mL}$ of concentrated hydrochloric acid were added into a $100 \mathrm{~mL}$ flask and mixed to get a heterogeneous solution under the atmosphere of nitrogen. Then, (3-Mercaptopropyl) trimethoxysilane $(8.5 \mathrm{~g}, 40 \mathrm{mmol})$ was added into the solution. The mixture was refluxed at $80^{\circ} \mathrm{C}$ for 5 days to produce white precipitates. The crude products were obtained after filtration, washing with cold methanol, and drying. Then recrystallization from acetone afforded the product as white solids with $18 \%$ yield. ${ }^{1} \mathrm{H} \mathrm{NMR}\left(\mathrm{CDCl}_{3}, 400 \mathrm{MHz}, \mathrm{ppm}\right): \delta 0.75(\mathrm{t}, 2 \mathrm{H})$, $1.35(\mathrm{~s}, 1 \mathrm{H}), 1.75(\mathrm{~m}, 2 \mathrm{H}), 2.53(\mathrm{q}, 2 \mathrm{H}) .{ }^{13} \mathrm{C} \mathrm{NMR}\left(\mathrm{CDCl}_{3}, 100 \mathrm{MHz}, \mathrm{ppm}\right)$ : $\delta 10.8,27.1,27.4 .{ }^{29} \mathrm{Si} \mathrm{NMR}\left(\mathrm{CDCl}_{3}, 300 \mathrm{MHz}, \mathrm{ppm}\right): \delta 34.9$.

PEG-SS-2TP polymer was synthesized as follows: PEG-SH $(10.2 \mathrm{~g}, 13.3 \mathrm{mmol})$ was dissolved in $50 \mathrm{~mL}$ of methanol and added dropwise to a stirred solution of 2 , $2^{\prime}$-dithiodipyridine $(8.8 \mathrm{~g}, 50 \mathrm{mmol})$ dissolved in $40 \mathrm{~mL}$ of methanol. The reaction was kept under an argon atmosphere to minimize free thiol oxidation. After 3 days, the mixture was concentrated under reduced pressure. The product was precipitated by addition of $50 \mathrm{~mL}$ of cold ether and purified by redissolving in $10 \mathrm{~mL}$ of methanol and precipitating into $50 \mathrm{~mL}$ of cold ether three times to give a white powder. The final product of PEG-SS-2TP was obtained in vacuo with $80 \%$ yield. ${ }^{1} \mathrm{H} \mathrm{NMR}\left(\mathrm{CDCl}_{3}, 400 \mathrm{MHz}, \mathrm{ppm}\right): \delta 2.99(\mathrm{t}, 2 \mathrm{H}), 3.37(\mathrm{~s}, 3 \mathrm{H}), 3.82(\mathrm{t}, 2 \mathrm{H})$, 7.08-8.45 (m, $4 \mathrm{H}) .{ }^{13} \mathrm{C} \mathrm{NMR}\left(\mathrm{CDCl}_{3}, 100 \mathrm{MHz}, \mathrm{ppm}\right): \delta 38.3,58.5,69.6,70.5,71.6$, $71.8,120.6,121.9,129.1,149.2159 .6$.

POSS-(SS-PEG) $)_{8}$ polymer was synthesized as follows: POSS- $(\mathrm{SH})_{8}(0.89 \mathrm{~g}$, $0.88 \mathrm{mmol}$ ) was dissolved in $50 \mathrm{~mL}$ of DCM and added dropwise to a stirred solution of PEG-SS-2TP $(9.31 \mathrm{~g}, 10.6 \mathrm{mmol})$ dissolved in $50 \mathrm{~mL}$ of DCM. The reaction was kept under nitrogen atmosphere to minimize free thiol oxidation. The reaction mixture was stirred at $40{ }^{\circ} \mathrm{C}$ for 6 days and then concentrated by rotary evaporation to yield a viscous liquid. The product of POSS-(SS-PEG) 8 was purified by ultrafiltration (MWCO 2000) and collected after freeze-drying with $68 \%$ yield. ${ }^{1} \mathrm{H} \mathrm{NMR}\left(\mathrm{CDCl}_{3}, 400 \mathrm{MHz}, \mathrm{ppm}\right): \delta 0.73(\mathrm{t}, 2 \mathrm{H}), 1.76(\mathrm{~m}, 2 \mathrm{H}), 2.41(\mathrm{t}, 2 \mathrm{H})$, $2.83(\mathrm{t}, 2 \mathrm{H}), 3.37$ (s, $3 \mathrm{H}) .{ }^{13} \mathrm{C} \mathrm{NMR}\left(\mathrm{CDCl}_{3}, 100 \mathrm{MHz}, \mathrm{ppm}\right): \delta 10.7,22.0,37.8$, $41.3,58.8,68.6,69.6,69.8,70.5,71.8$.

Synthesis of hyperbranched poly(BAC2-AEPZ1)-PEG polymer. AEPZ (0.4 g, $3 \mathrm{mmol}$ ) was dissolved in $5 \mathrm{~mL}$ of methanol and dropwise added into $20 \mathrm{~mL}$ of methanol containing the BAC $(1.58 \mathrm{~g}, 6.0 \mathrm{mmol})$. The solution was stirring at $50^{\circ} \mathrm{C}$ for 6 days, and then PEG-NH 
stirring at $60^{\circ} \mathrm{C}$ for another 5 days to seal terminal vinyl groups. The reaction mixture was concentrated by rotary evaporation and precipitated in $100 \mathrm{~mL}$ of cold ether for three times. Thereafter, the target polymer was collected and purified by redissolving in $10 \mathrm{~mL}$ of methanol and precipitating into $200 \mathrm{~mL}$ of acetone containing $10 \mathrm{~mL}$ of $37 \%$ concentration $\mathrm{HCl}$. The final product of poly(BAC2AEPZ1)-PEG polymer $\left(M_{\mathrm{n}}=1.9 \mathrm{kDa}, \mathrm{PDI}=2.0\right)$ was obtained in vacuo at $50^{\circ} \mathrm{C}$ overnight.

Synthesis of multi-arm CD-(SS-PEG) $)_{\mathbf{3}-4}$ polymer. $\beta$-CD $(1.14 \mathrm{~g}, 1 \mathrm{mmol})$ and $\mathrm{Et}_{3} \mathrm{~N}(0.7 \mathrm{~mL}, 5 \mathrm{mmol})$ was dissolved in $25 \mathrm{~mL}$ of dry DMF and added dropwise to a stirred solution of PEG-SS-COCl (3.2 g, $4 \mathrm{mmol})$ dissolved in $20 \mathrm{~mL}$ of DMF. The reaction mixture was stirred at $40^{\circ} \mathrm{C}$ for $6 \mathrm{~h}$ and then concentrated by rotary evaporation to yield a viscous liquid. The product, CD-(SS-PEG) $(n=3-4,3.15 \mathrm{~g}$, $80 \%$ ), was purified by ultrafiltration (MWCO 2000) and collected after freezedrying.

pH-triggered morphology and size evolution in water. A total of $5 \mathrm{mg}$ of POSS(SS-PEG) ${ }_{8}$ was dissolved in $5 \mathrm{~mL}$ of deionized water with $10 \mu \mathrm{L}$ of $5 \mathrm{M} \mathrm{NaOH}$ was added to adjust $\mathrm{pH}$ to 12 or 8 . Then $0.5 \mathrm{mg}$ of cysteamine was mixed to trigger the reaction. After standing for a certain time, the stable morphology can be obtained by neutralization with $0.2 \mathrm{~mL}$ of saturated $\mathrm{NH}_{4} \mathrm{Cl}$ solution, and the morphology evolution was obtained from the solution samples at a predetermined time. Similar to the above preparation process for POSS-(SS-PEG) $)_{8}$ polymer with the concentration of $0.1 \mathrm{mg} / \mathrm{mL}$ in $\mathrm{pH} 12$ solutions, its morphology evolution was also obtained from the solution samples at a predetermined time.

The self-assembly process of hyperbranched poly(BAC2-AEPZ1)-PEG and CD$(\mathrm{SS}-\mathrm{PEG})_{3-4}$ polymers were as same as that of POSS-(SS-PEG) 8 polymer with the concentration of $1 \mathrm{mg} / \mathrm{mL}$ in $\mathrm{pH} 12$ solutions, After standing for a certain time, the stable morphology can be obtained by neutralization with $0.2 \mathrm{~mL}$ of saturated $\mathrm{NH}_{4} \mathrm{Cl}$ solution, and the morphology evolution was obtained from the solution samples at a predetermined time.

pH-triggered morphology and size evolution in THF/water. A total of $5 \mathrm{mg}$ of POSS-(SS-PEG) ${ }_{8}$ was dissolved in $5 \mathrm{~mL}$ of THF/water $(v / v=1: 4)$ with $10 \mu \mathrm{L}$ of $5 \mathrm{M}$ $\mathrm{NaOH}$ was added to adjust $\mathrm{pH}$ to 12 . Then $0.5 \mathrm{mg}$ of cysteamine was mixed to trigger the reaction. After standing for a certain time, the stable morphology can be obtained by neutralization with $0.2 \mathrm{~mL}$ of saturated $\mathrm{NH}_{4} \mathrm{Cl}$ solution and morphology evolution was obtained from the solution samples at a predetermined time.

UV-triggered morphology and size evolution in water. A total of $5 \mathrm{mg}$ of POSS(SS-PEG) ${ }_{8}$ was dissolved in $5 \mathrm{~mL}$ of deionized water with $0.5 \mathrm{mg}$ of cysteamine and $0.1 \mathrm{mg}$ of IR2959. Then irradiation triggered the reaction under a UV lamp $(300 \mathrm{~W}, 200-400 \mathrm{~nm})$ with gentle rotation by using a mechanical agitator to ensure homogeneity. After standing for a certain time, the stable morphology can be obtained by removal of the UV irradiation, and the morphology evolution was obtained from the solution samples at a predetermined irradiation time.

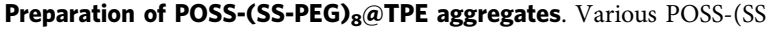
$\mathrm{PEG})_{8} @ \mathrm{TPE}$ aggregates were prepared as follows: $5 \mathrm{mg}$ of POSS-(SS-PEG) ${ }_{8}$ and $1 \mathrm{mg}$ of TPE-CHO molecules were firstly dissolved in $1 \mathrm{~mL}$ of THF, then $4 \mathrm{~mL}$ of ultrapure water was added dropwise into the solution at the rate of $0.05 \mathrm{~mL} / \mathrm{min}$ via a syringe pump. Then $10 \mu \mathrm{L}$ of $5 \mathrm{M} \mathrm{NaOH}$ was added to adjust $\mathrm{pH}$ to 12 . Then $0.5 \mathrm{mg}$ of cysteamine was mixed to trigger the reaction. After standing for a certain time, the stable morphology can be obtained by neutralization with $0.2 \mathrm{~mL}$ of saturated $\mathrm{NH}_{4} \mathrm{Cl}$ solution, and the morphology evolution was obtained from the solution samples at a predetermined time. Then the photograph, CLSM image and fluorescent intensity of various TPE-loaded aggregates were measured by fluorescence measurements.

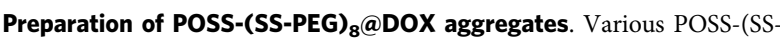
PEG) $)_{8} @ D O X$ aggregates were prepared as follows: $5 \mathrm{mg}$ of POSS-(SS-PEG) 8 was dissolved in $1 \mathrm{~mL}$ of DMF, followed by adding a predetermined amount of $\mathrm{DOX} \cdot \mathrm{HCl}$ and 1.5 molar equiv of triethylamine and stirred at room temperature for $2 \mathrm{~h}$. Then $4 \mathrm{~mL}$ of PBS ( $\mathrm{pH} \mathrm{7.4,8,} \mathrm{or} \mathrm{12)} \mathrm{was} \mathrm{added} \mathrm{dropwise} \mathrm{into} \mathrm{the} \mathrm{solution}$ at the rate of $0.05 \mathrm{~mL} / \mathrm{min}$ via a syringe pump. After triggering the exchange reaction for a certain time, the stable morphology can be obtained by neutralization with saturated $\mathrm{NH}_{4} \mathrm{Cl}$ solution and morphology evolution was obtained from the solution samples at a predetermined time. Subsequently, the solution was dialyzed against deionized water for $24 \mathrm{~h}(\mathrm{MW}$ cutoff, $4 \mathrm{kDa}$ ) to remove free DOX and byproducts, followed by lyophilization to obtain the freeze various dried DOX-loaded aggregates.

In vitro DOX release. A series of DOX-loaded POSS-(SS-PEG) 8 aggregates with various thiol-disulfide exchange reaction times were added into a dialysis membrane tube (MW cutoff, $4 \mathrm{kDa}$ ) and then immersed into $30 \mathrm{~mL}$ of $\mathrm{pH} 7.4$ PBS containing $10 \mathrm{mM}$ DTT in a shaking water bath at $37^{\circ} \mathrm{C}$. The POSS-(SS-

$\mathrm{PEG})_{8} @ \mathrm{DOX}$ solutions were lyophilized and then dissolved in DMF to determine the total loading of DOX by the fluorescence spectroscopy. The amount of DOX release was determined using UV-vis absorbance (excitation at $480 \mathrm{~nm}$ ). All the DOX release experiments were conducted in triplicate and the results were expressed as the average \pm standard deviation.

Cellular uptake and intracellular localization. MCF-7 cells were seeded onto a 96-well plate at a density of $5 \times 10^{4}$ cells/well in Dulbecco's Modified Eagle Medium (DMEM) containing 10\% fetal bovine serum (FBS). After incubation for $24 \mathrm{~h}$ $\left(37^{\circ} \mathrm{C}, 5 \% \mathrm{CO}_{2}\right)$, the medium was removed and replenished by the fresh DMEM containing $10 \%$ FBS. Then the prescribed amounts of free DOX, POSS-(SSPEG) $)_{8} @ D O X-8$ h, and POSS-(SS-PEG) $)_{8} @ D O X-36$ h aggregates were added. After the incubation of cells for various times, the culture medium was removed and the cells were washed with PBS for several times. Afterwards, the cells were fixed with $4 \%$ paraformaldehyde at room temperature and the slides were rinsed with PBS for three times. Finally, the MCF-7 cells were directly observed using a confocal laser scanning microscopy (CLSM) with the excitation of $488 \mathrm{~nm}$.

Activity analyses. The cytotoxicity of POSS-(SS-PEG) $)_{8}$ and POSS-(SS-PEG) $)_{8} @$ DOX aggregates (exchange reaction at $\mathrm{pH} 7.4$ for 8 and $36 \mathrm{~h}$ ) was evaluated in vitro by CCK- 8 assay. MCF-7 cells were seeded in $180 \mu \mathrm{L}$ of DMEM containing $10 \%$ FBS at a density of $1 \times 10^{4}$ cells/well. After incubation for $24 \mathrm{~h}\left(37^{\circ} \mathrm{C}, 5 \% \mathrm{CO}_{2}\right)$, the medium was replaced by $90 \mu \mathrm{L}$ of fresh DMEM containing $10 \% \mathrm{FBS}$, and then $20 \mu \mathrm{L}$ of samples with various concentrations of nanoparticle suspensions in PBS $(\mathrm{pH}$ 7.4) were added and further incubated for another $24 \mathrm{~h}$. After removal of the culture medium, $100 \mu \mathrm{L}$ of fresh DMEM and $10 \mu \mathrm{L}$ of CCK-8 kit solutions were immediately added and homogeneously mixed. After incubating the cells for $4 \mathrm{~h}$ in a $\mathrm{CO}_{2}$ incubator, $100 \mu \mathrm{L}$ of reaction solutions were put into a 96 -well plate. The optical density of each well at $450 \mathrm{~nm}$ was carefully measured by a microplate reader. Cells cultured in DMEM containing 10\% FBS without pretreatment were used as the control. The resulting data were expressed as the average \pm standard deviation $(n=5)$.

All-atom MD simulations. All the simulations were carried out using GROMACS The force field of POSS ${ }_{m}-\mathrm{PEG}_{n}$ oligomer were from refs. ${ }^{62,63}$. The TIP3P water model was used for water. In MD simulations, one POSS ${ }_{m}-\mathrm{PEG}_{n}$ oligomer with different degree of polymerization was solvated in water box. The details of box size are listed in Supplementary Table 1 . These systems were firstly simulated at $300 \mathrm{~K}$ and $1 \mathrm{~atm}$ for $20 \mathrm{~ns}$. Then $1 \mu$ s production simulations were performed. The time step is $2 \mathrm{fs}$. The temperature and pressure were maintained by berendsen thermostat and barostat in equilibrium and in production simulation the Nose-Hoover thermostat and Parrinello-Rahman barostat were used. Electrostatic interactions were calculated using particle mesh Ewald and a real space cutoff of $1.2 \mathrm{~nm}$. van der Waals interactions were switched to zero between $1 \mathrm{~nm}$ and $1.2 \mathrm{~nm}$. Three independent MD runs were conducted for every system.

DPD simulations. To understand the self-assembly behaviors of POSS-(SS-PEG) polymer in water, some DPD simulations were performed by constructing a specific coarse-grained (CG) model of POSS-(SS-PEG) ${ }_{8}$ polymer wherein the eight branches were attached to one big CG POSS bead. As shown in Supplementary Fig. 8, there were two types of beads, i.e., P and G for POSS and PEG. The atomistic model was coarse-grained according to the molecular segment size in all-atom simulation. In this study we adjusted the size of central POSS bead to simulate the polymerization of POSS-domain cores. The interaction parameter $\mathrm{a}_{\mathrm{ij}}$ between POSS and PEG beads was also increased to describe the dynamic hydrophobic interaction.

Within the CG model, neighboring CG beads are bonded to each other by a harmonic spring potential, $U_{\mathrm{b}}=0.5 k_{\mathrm{b}}\left(r-r_{0}\right)^{2}$, where $k_{\mathrm{b}}$ is the spring constant and $r_{0}$ is the equilibrium bond length. In this study, $k_{\mathrm{b}}=25$ and $r_{0}=0.7$. The concentration of POSS-(SS-PEG) ${ }_{8}$ polymer in the solution $\varphi$ is 0.05 , which is calculated by the following formula,

$$
\varphi=\frac{N_{\mathrm{P}} V_{\mathrm{P}}+N_{\mathrm{G}} V_{\mathrm{G}}}{N_{\mathrm{P}} V_{\mathrm{P}}+N_{\mathrm{G}} V_{\mathrm{G}}+N_{\mathrm{W}} V_{\mathrm{W}}}
$$

where $N_{\mathrm{P}}, N_{\mathrm{G}}$, and $N_{\mathrm{W}}$ are the number of POSS, PEG, and water beads while $V_{\mathrm{P}}$, $V_{\mathrm{G}}$, and $V_{\mathrm{W}}$ are the volume of one bead of POSS, PEG, and solvent, respectively. The simulations were performed in NVT ensemble with periodical boundary conditions and at a fixed system number density of 3.0. All the CG beads have the same mass as $m=1$. The interaction cutoff radius is set to 1 as the unit of length The reduced temperature is 1.0 . The size of simulation box is $30 \times 30 \times 30$. In the DPD simulations the modified velocity-verlet algorithm was used to integrate the equations of motion with a time step of 0.03 . The simulation systems were preequilibrated with $1 \times 10^{6}$ steps by setting $a_{i j}=25$ and $T=1.0$ for all DPD beads. Then we performed another simulation with $2 \times 10^{6}$ steps to observe the morphology evolution of aggregates.

General characterization. ${ }^{1} \mathrm{H},{ }^{13} \mathrm{C}$, and ${ }^{29} \mathrm{Si}$ NMR spectra were recorded on a Bruker DRX-400 spectrometer using deuterated chloroform $(\mathrm{CDCl} 3)$ as the solvent and tetramethylsilane (TMS) as the internal standard. Transmission electron microscopy (TEM) studies were performed with a JEM-2200FS instrument (JEOL 
Japan). Cryogenic transmission electron microscopy (Cryo-TEM) studies were examined in a cryogenic sample holder (Gatan 626) with a JEM-2200FS at approximately $-174^{\circ} \mathrm{C}$. Scanning electron microscopy (SEM) images were recorded on a JSM-6700F microscope (JEOL, Japan) with the acceleration voltage of $5 \mathrm{kV}$. Atomic force microscope (AFM) images were obtained with a Nanoscope Multimode III AFM instrument (Vecco, America) in tapping mode. Dynamic light scattering (DLS) profiles were measured on a commercial laser light scattering spectrometer (ALV/DLS/SLS-5022F) equipped with a multi- $\tau$ digital time correlator (ALV5000) and a cylindrical $22 \mathrm{~mW}$ UNIPHASE He-Ne laser $\left(\lambda_{0}=632.8 \mathrm{~nm}\right)$. Confocal laser scanning microscopy (CLSM, Zeiss LSM 510, Germany) images were obtained under the excitation at $488 \mathrm{~nm}$. UV-vis spectra were recorded by a Hitachi U-3010 spectrometer and fluorescence measurements were performed on a Hitachi F4600 photoluminescence spectrometer with a xenon lamp as a light source.

Data availability. The authors declare that the data supporting the conclusions of this study are available within the article and its Supplementary Information file or from the corresponding author upon reasonable request.

Received: 27 November 2017 Accepted: 11 June 2018

Published online: 17 July 2018

\section{References}

1. Kelley, E. G. et al. Size evolution of highly amphiphilic macromolecular solution assemblies via a distinct bimodal pathway. Nat. Commun. 5, 3599 (2014).

2. El Garah, M. et al. Atomically precise prediction of 2D self-assembly of weakly bonded nanostructures: STM insight into concentration-dependent architectures. Small 12, 343-350 (2016).

3. Stefik, M., Guldin, S., Vignolini, S., Wiesner, U. \& Steiner, U. Block copolymer self-assembly for nanophotonics. Chem. Soc. Rev. 44, 5076-5091 (2015).

4. Palivan, C. G. et al. Bioinspired polymer vesicles and membranes for biological and medical applications. Chem. Soc. Rev. 45, 377-411 (2016).

5. Bishop, K. J. M. Hierarchical self-assembly for nanomedicine. Angew. Chem. Int. Ed. 55, 1598-1600 (2016).

6. Zhu, X. et al. Polymeric nanoparticles amenable to simultaneous installation of exterior targeting and interior therapeutic proteins. Angew. Chem. Int. Ed. 55, 3309-3312 (2016).

7. Mai, Y. Y. \& Eisenberg, A. Self-assembly of block copolymers. Chem. Soc. Rev. 41, 5969-5985 (2012).

8. Li, J. W., Carnall, J. M. A., Stuart, M. C. A. \& Otto, S. Hydrogel formation upon photoinduced covalent capture of macrocycle stacks from dynamic combinatorial libraries. Angew. Chem. Int. Ed. 50, 8384-8386 (2011).

9. Zhang, J. Y. et al. Antimicrobial metallopolymers and their bioconjugates with conventional antibiotics against multidrug-resistant bacteria. J. Am. Chem. Soc. 136, 4873-4876 (2014).

10. Pal, A. et al. Controlling the structure and length of self-synthesizing supramolecular polymers through nucleated growth and disassembly. Angew. Chem. Int. Ed. 54, 7852-7856 (2015).

11. Corbett, P. T. et al. Dynamic combinatorial chemistry. Chem. Rev. 106, 3652-3711 (2006).

12. Versluis, F., van Esch, J. H. \& Eelkema, R. Synthetic self-assembled materials in biological environments. Adv. Mater. 28, 4576-4592 (2016).

13. Wang, X. et al. Fabrication of $\mathrm{pH}$-responsive nanoparticles with an AIE feature for imaging intracellular drug delivery. Biomacromolecules 17 2920-2929 (2016).

14. Ma, N., Li, Y., Xu, H. P., Wang, Z. Q. \& Zhang, X. Dual redox responsive assemblies formed from diselenide block copolymers. J. Am. Chem. Soc. 132, 442-443 (2010).

15. Bell, O. A. et al. Self-assembly of a functional oligo(aniline)-based amphiphile into helical conductive nanowires. J. Am. Chem. Soc. 137, 14288-14294 (2015).

16. Li, Z., Ma, J., Lee, N. S. \& Wooley, K. L. Dynamic cylindrical assembly of triblock copolymers by a hierarchical process of covalent and supramolecular interactions. J. Am. Chem. Soc. 133, 1228-1231 (2011).

17. Liu, F., Lehn, J. M. \& Hirsch, A. K. H. Molecular biodynamers: dynamic covalent analogues of biopolymers. Acc. Chem. Res. 50, 376-386 (2017).

18. Tan, K. W. et al. Transient laser heating induced hierarchical porous structures from block copolymer-directed self-assembly. Science 349, 54-58 (2015).

19. Wang, Y. P., Xu, H. P. \& Zhang, X. Tuning the amphiphilicity of building blocks: controlled self-assembly and disassembly for functional supramolecular materials. Adv. Mater. 21, 2849-2864 (2009).

20. Valle, K., Belleville, P., Pereira, F. \& Sanchez, C. Hierarchically structured transparent hybrid membranes by in situ growth of mesostructured organosilica in host polymer. Nat. Mater. 5, 107-111 (2006).
21. Frisch, H. et al. Kinetically controlled sequential growth of surface-grafted chiral supramolecular copolymers. Angew. Chem. Int. Ed. 55, 7242-7246 (2016).

22. Wang, C., Chen, Q. S., Xu, H. P., Wang, Z. Q. \& Zhang, X. Photoresponsive supramolecular amphiphiles for controlled self-assembly of nanofibers and vesicles. Adv. Mater. 22, 2553-2555 (2010).

23. Mason, T. O. et al. Synthesis of nonequilibrium supramolecular peptide polymers on a microfluidic platform. J. Am. Chem. Soc. 138, 9589-9596 (2016).

24. Wang, C. et al. Controlled self-assembly manipulated by charge-transfer interactions: from tubes to vesicles. Angew. Chem. Int. Ed. 47, 9049-9052 (2008).

25. Gorgoll, R. M., Harano, K. \& Nakamura, E. Nanoscale control of polymer assembly on a synthetic catalyst-bilayer system. J. Am. Chem. Soc. 138, 9675-9681 (2016).

26. Wang, P. F. et al. Programming self-assembly of DNA origami honeycomb two-dimensional lattices and plasmonic metamaterials. J. Am. Chem. Soc. 138, 7733-7740 (2016)

27. Li, D. W. et al. Synthesis and self-assembly behavior of POSS-embedded hyperbranched polymers. Chem. Commun. 51, 8296-8299 (2015).

28. Hunt, S. T. et al. Self-assembly of noble metal monolayers on transition metal carbide nanoparticle catalysts. Science 352, 974-978 (2016).

29. Huang, M. J. et al. Selective assemblies of giant tetrahedra via precisely controlled positional interactions. Science 348, 424-428 (2015).

30. Groschel, A. H. et al. Guided hierarchical co-assembly of soft patchy nanoparticles. Nature 503, 247-251 (2013).

31. Ji, X. F. et al. Controlling amphiphilic copolymer self-assembly morphologies based on macrocycle/anion recognition and nucleotide-induced payload release. Chem. Sci. 7, 6006-6014 (2016).

32. Chakrabarty, R., Mukherjee, P. S. \& Stang, P. J. Supramolecular coordination: self-assembly of finite two-and three-dimensional ensembles. Chem. Rev. 111, 610-6818 (2011).

33. Iha, R. K. et al. Applications of orthogonal "click" chemistries in the synthesis of functional soft materials. Chem. Rev. 109, 5620-5686 (2009).

34. Hawker, C. J. et al. The convergence of synthetic organic and polymer chemistries. Science 309, 1200-1205 (2005).

35. Elsabahy, M. \& Wooley, K. L. Design of polymeric nanoparticles for biomedical delivery applications. Chem. Soc. Rev. 41, 2545-2561 (2012).

36. Moridi, N., Corvini, P. F. X. \& Shahgaldian, P. Reversible supramolecular surface attachment of enzyme-polymer conjugates for the design of biocatalytic filtration membranes. Angew. Chem. Int. Ed. 54, 14800-14804 (2015).

37. McKeown, J. T., Wu, Y. Y., Fowlkes, J. D., Rack, P. D. \& Campbell, G. H. Simultaneous in-situ synthesis and characterization of Co@Cu core-shell nanoparticle arrays. Adv. Mater. 27, 1060-1065 (2015).

38. Chi, X. D. et al. A dual-thermoresponsive gemini-type supra-amphiphilic macromolecular [3]pseudorotaxane based on pillar[10] arene/paraquat cooperative complexation. J. Am. Chem. Soc. 138, 3168-3174 (2016).

39. Youssef, M., Theodore, H., Yi, G. R. \& Sacanna, S. Shape-shifting colloids via stimulated dewetting. Nat. Commun. 7, 12216 (2016).

40. Cui, H. G. et al. Block copolymer assembly via kinetic control. Science 317, 647-650 (2007)

41. Chandrasekhar, N. \& Chandrasekar, R. Reversibly shape-shifting organic optical waveguides: formation of organic nanorings, nanotubes, and nanosheets. Angew. Chem. Int. Ed. 51, 3556-3561 (2016).

42. Cai, C. H. et al. Simulation-assisted self-assembly of multicomponent polymers into hierarchical assemblies with varied morphologies. Angew. Chem. Int. Ed. 52, 7732-7736 (2013).

43. Zhuang, Z. L. et al. Hierarchical nanowires synthesized by supramolecular stepwise polymerization. Angew. Chem. Int. Ed. 55, 1-7 (2016).

44. Han, Y. K. et al. An intriguing morphology evolution of polyoxometalatepolystyrene hybrid amphiphiles from vesicles to tubular aggregates. Macromol. Chem. Phys. 212, 81-87 (2011).

45. Ma, C. et al. A Filled-honeycomb-structured crystal formed by self-assembly of a Janus polyoxometalate-silsesquioxane (POM-POSS) co-cluster. Angew. Chem. Int. Ed. 54, 15699-15704 (2015).

46. Wu, D. C., Loh, X. J., Wu, Y. L., Lay, C. L. \& Liu, Y. 'Living' controlled in situ gelling systems: thiol-disulfide exchange method toward tailor-made biodegradable hydrogels. J. Am. Chem. Soc. 132, 15140-15143 (2010).

47. Wang, X. et al. Controlled cross-linking strategy: from hybrid hydrogels to nanoparticle macroscopic aggregates. Polym. Chem. 4, 4596-4600 (2013).

48. Yu, X. F. et al. A giant surfactant of polystyrene-(carboxylic acidfunctionalized polyhedral oligomeric silsesquioxane) amphiphile with highly stretched polystyrene tails in micellar assemblies. J. Am. Chem. Soc. 132, 16741-16744 (2010).

49. Li, Y. W. et al. Breaking symmetry toward nonspherical Janus particles based on polyhedral oligomeric silsesquioxanes: molecular design, "click" synthesis, and hierarchical structure. J. Am. Chem. Soc. 133, 10712-10715 (2011). 
50. Ni, B. et al. Pathway toward large two-dimensional hexagonally patterned colloidal nanosheets in solution. J. Am. Chem. Soc. 137, 1392-1395 (2015).

51. Wang, X. et al. Facile creation of FRET systems from a pH-responsive AIE fluorescent vesicle. Chem. Commun. 52, 5320-5323 (2016).

52. Wang, X. et al. Synthesis, self-assembly, and photoresponsive behavior of tadpole-shaped azobenzene polymers. ACS Macro Lett. 4, 1321-1326 (2015).

53. Wang, X. et al. POSS dendrimers constructed from a $1 \rightarrow 7$ branching monomer. Chem. Commun. 50, 6126-6129 (2014).

54. Dong, X. H. et al. Molecular-curvature-induced spontaneous formation of curved and concentric lamellae through nucleation. Angew. Chem. Int. Ed. 55, 2459-2463 (2016).

55. Wu, K. et al. Asymmetric giant "bolaform-like" surfactants: precise synthesis, phase diagram, and crystallization-induced phase separation. Macromolecules 47, 4622-4633 (2014).

56. Liu, H. et al. Manipulation of self-assembled nanostructure dimensions in molecular Janus particles. ACS Nano 10, 6585-6596 (2016).

57. Zhang, W. A. \& Müller, A. H. E. Architecture, self-assembly and properties of well-defined hybrid polymers based on polyhedral oligomeric silsequioxane (POSS). Prog. Polym. Sci. 38, 1121-1162 (2013).

58. Ma, S. Y., Hu, Y. \& Wang, R. Self-assembly of polymer tethered molecular nanoparticle shape amphiphiles in selective solvents. Macromolecules 48, 3112-3120 (2015).

59. Hoogerbrugge, P. J. \& Koelman, J. M. V. A. Simulating microscopic hydrodynamic phenomena with dissipative particle dynamics. Europhys. Lett. 19, 155-160 (1992).

60. Koelman, J. M. V. A. \& Hoogerbrugge, P. J. Dynamic simulations of hardsphere suspensions under steady shear. Europhys. Lett. 21, 363-368 (1993).

61. Liu, Y. et al. A supramolecular Janus hyperbranched polymer and its photoresponsive self-assembly of vesicles with narrow size distribution. J. Am. Chem. Soc. 135, 4765-4770 (2013).

62. Lee, H., de Vries, A. H., Marrink, S. J. \& Pastor, R. W. A coarse-grained model for polyethylene oxide and polyethylene glycol: conformation and hydrodynamics. J. Phys. Chem. B 113, 13186-13194 (2009).

63. Neyertz, S., Brown, D., Raaijmakers, M. J. T. \& Benes, N. E. The influence of the dianhydride precursor in hyper-cross-linked hybrid polyPOSS-imide networks. Phys. Chem. Chem. Phys. 18, 28688-28703 (2016).

\section{Acknowledgements}

This work was supported by NSFC (Nos. 21504096, 21725403, 21674120, and 21474115), the Ministry of Science and Technology of China (No. 2014CB932200), and "Young
Thousand Talents Program”. P.G. was supported by the U.S. Department of Energy, Office of Science, and Office of Advanced Scientific Computing Research, as part of the Collaboratory on Mathematics for Mesoscopic Modelling of Materials (CM4). Pacific Northwest National Laboratory is operated by Battelle for the DOE under Contract No. DE-AC05-76RL01830.

\section{Author contributions}

X.W. and D.W. designed the whole work and wrote the manuscript. X.W. carried out the experiments and analyzed data. Y.Y. performed the characterization and analyzed data. P.G. and H.G. designed and carried out the simulations and related analysis.

\section{Additional information}

Supplementary Information accompanies this paper at https://doi.org/10.1038/s41467018-05142-3.

Competing interests: The authors declare no competing interests.

Reprints and permission information is available online at http://npg.nature.com/ reprintsandpermissions/

Publisher's note: Springer Nature remains neutral with regard to jurisdictional claims in published maps and institutional affiliations.

(c) (i) Open Access This article is licensed under a Creative Commons Attribution 4.0 International License, which permits use, sharing, adaptation, distribution and reproduction in any medium or format, as long as you give appropriate credit to the original author(s) and the source, provide a link to the Creative Commons license, and indicate if changes were made. The images or other third party material in this article are included in the article's Creative Commons license, unless indicated otherwise in a credit line to the material. If material is not included in the article's Creative Commons license and your intended use is not permitted by statutory regulation or exceeds the permitted use, you will need to obtain permission directly from the copyright holder. To view a copy of this license, visit http://creativecommons.org/ licenses/by/4.0/.

(C) The Author(s) 2018 